\title{
Generalizing Negative Imaginary Systems Theory to Include Free Body Dynamics: Control of Highly Resonant Structures with Free Body Motion
}

\author{
M. A. Mabrok, A. G. Kallapur, I. R. Petersen and A. Lanzon
}

\begin{abstract}
Index Terms-Negative imaginary systems, flexible structures, free body motion.

Abstract-Negative imaginary (NI) systems play an important role in the robust control of highly resonant flexible structures. In this paper, a generalized NI system framework is presented. A new NI system definition is given, which allows for flexible structure systems with colocated force actuators and position sensors, and with free body motion. This definition extends the existing definitions of NI systems. Also, necessary and sufficient conditions are provided for the stability of positive feedback control systems where the plant is NI according to the new definition and the controller is strictly negative imaginary. The stability conditions in this paper are given purely in terms of properties of the plant and controller transfer function matrices, although the proofs rely on state space techniques. Furthermore, the stability conditions given are independent of the plant and controller system order. As an application of these results, a case study involving the control of a flexible robotic arm with a piezoelectric actuator and sensor is presented.
\end{abstract}

\section{INTRODUCTION}

Flexible structure dynamics arise in many areas such as flexible robot manipulators [1], ground and aerospace vehicles [2], atomic force microscopes (AFMs) [3], [4] and other nano-positioning systems [5]-[8]. Flexible structures can be modeled as infinite dimensional distributed parameter systems [9]. However, finite dimensional models are often used for the purpose of designing controllers [9]-[12]. In designing controllers for these flexible systems, it is important to consider the effect of highly resonant modes. Such resonant modes are known to adversely affect the stability and performance of flexible structure feedback control systems [12]-[14], and are often very sensitive to changes in environmental variables. For instance, a small change in the environment of the system such as changing temperature, can lead to significant changes in the resonant frequencies of such systems. These changes in resonant frequencies can lead to large changes in the gain and

M. Mabrok, A. Kallapur and I. R. Petersen are with the School of Engineering and Information Technology, University of New South Wales at the Australian Defence Force Academy, Canberra ACT 2600, Australia, email:abdallamath@gmail.com, abhijit.kallapur@gmail.com, i.r.petersen@gmail.com.

A. Lanzon is with the Control Systems Centre, School of Electrical and Electronic Engineering, University of Manchester, Manchester M13 9PL, United Kingdom, email:Alexander.Lanzon@manchester.ac.uk.

This research was supported by the Australian Research Council and the EPSRC. phase of the system frequency response at a given frequency, which may lead to instability or poor performance in the corresponding feedback system. In addition, highly resonant modes lead to vibrational effects which limit the ability of control systems to achieve desired levels of performance in many applications such as precision instrumentation, optical systems, precision machine tools, wafer steppers, telescopes, and atomic force microscopes [12]. These issues arising from the presence of highly resonant modes in flexible structures motivate the need for tools to guarantee robust stability and performance in flexible structure control systems.

One common solution to issues of robustness, stability, and performance in the control of highly resonant flexible structures is to use force actuators combined with colocated measurements of velocity, position, or acceleration [12]-[14]. Colocated control with velocity measurements, known as negative-velocity feedback, can be used to directly increase the effective damping in the system, thereby facilitating the design of controllers that can guarantee closed-loop stability in the presence of parameter variations and unmodeled plant dynamics [12]. Similarly, a class of colocated controllers with position measurements, known as positive-position feedback controllers, where velocity sensors are replaced with position sensors, can also be used to increase damping in flexible systems as discussed in [13], [15]. Also, positive-position feedback controllers are robust against uncertainties in resonant frequencies as well as unmodeled plant dynamics, in a similar way to negative-velocity feedback controllers [13], [14], [16].

The properties of negative-velocity feedback has been studied using passivity theory and the theory of positive real (PR) linear time invariant (LTI) systems; e.g., see [17], [18]. However, PR theory cannot be used directly when using position or acceleration measurements [14]. This drawback is important in applications to the field of nanotechnology, especially for nano-positioning systems, where position measurements are widely used; see e.g., [3], [5]-[8], [19]-[23]. Similar issues also arise in application to the area of robotics where position measurements are also widely used.

Lanzon and Petersen introduced a notion of negative imaginary (NI) systems in [14], [16] for the robust control of flexible structures with force actuators combined with position or acceleration sensors. (SISO) case, NI systems are defined by considering the properties of the imaginary part of the system frequency response $G(j \omega)$ and requiring the condition 


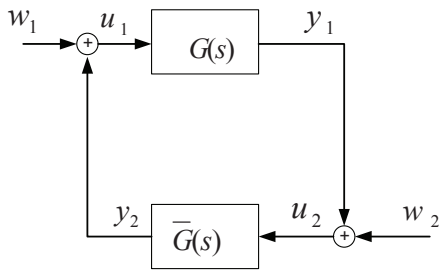

Fig. 1. A negative-imaginary feedback control system. If the plant transfer function matrix $G(s)$ is NI and the controller transfer function matrix $\bar{G}(s)$ is SNI, then the positive-feedback interconnection is internally stable if and only if the DC gain condition, $\lambda_{\max }(G(0) \bar{G}(0))<1$, is satisfied.

$j\left(G(j \omega)-G(j \omega)^{*}\right) \geq 0$ for all $\omega \in(0, \infty)$. The NI property arises in many practical systems. For example, such systems arise when considering the transfer function from a force actuator to a corresponding colocated position sensor (for instance, a piezoelectric sensor) in a lightly damped structure [3], [13], [14], [24], [25]. Another area where the underlying system dynamics are NI, in the area of nano-positioning systems; see e.g., [3], [5]-[8], [19]-[23]. Also, the positive-position feedback control scheme in [13], [26], can be considered using the NI framework. Furthermore, other control methodologies in the literature such as integral resonant control (IRC) [27] and resonant feedback control [28], [29], fit into the NI framework and their stability robustness properties can be explained by NI systems theory.

The stability robustness of interconnected NI systems has been studied in [14], [16]. In these papers, it is shown that a necessary and sufficient condition for the internal stability of a positive-feedback control system (see Fig. 1) consisting of an NI plant with transfer function matrix $G(s)$ and a strictly negative imaginary (SNI) controller with transfer function matrix $\bar{G}(s)$ is given by the DC gain condition

$$
\lambda_{\max }(G(0) \bar{G}(0))<1,
$$

where the notation $\lambda_{\max }(\cdot)$ denotes the maximum eigenvalue of a matrix with only real eigenvalues. This stability result has been used in a number of practical applications [3], [4], [8], [25], [30], [31]. For example in [25], this stability result is applied to the problem of decentralized control of large vehicle platoons. In [3], [4], the NI stability result is applied to nanopositioning in an atomic force microscope. A positive position feedback control scheme based on the NI stability result provided in [14], [16] is used to design a novel compensation method for a coupled fuselage-rotor mode of a rotary wing unmanned aerial vehicle in [30]. In [8], an IRC scheme based on the stability results provided in [14], [16] is used to design an active vibration control system for the mitigation of human induced vibrations in light-weight civil engineering structures, such as floors and footbridges via proof-mass actuators. An identification algorithm which enforces the NI constraint is proposed in [31] for estimating model parameters, following which an Integral resonant controller is designed for damping vibrations in flexible structures. In addition, it is shown in [32] that the class of linear systems having NI transfer function matrices is closely related to the class of linear Hamiltonian input-output systems. Also, an extension of the NI systems

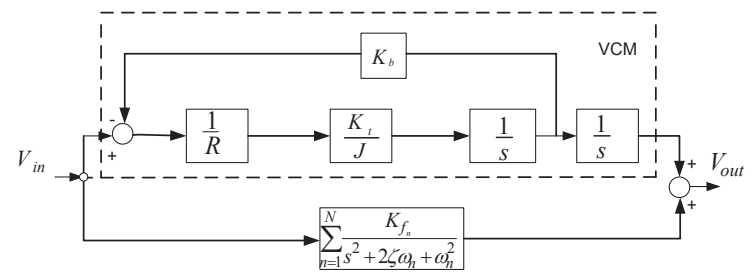

Fig. 2. Block diagram of a disk-drive reader head system which includes a flexible structure driven by a voice coil motor (VCM). The parameters $R, K_{t}, J$, and $K_{b}$ are the coil resistance, torque constant, moment of inertia, and back electromotive force gain, respectively. Also, the parameters $K_{f}, \zeta$, and $\omega_{n}$ are the flexible structure gain, damping ratio, and natural frequency respectively.

theory to infinite-dimensional systems is presented in [33].

The NI framework presented in [14], [16] considers systems with poles in the open left half of the complex plane. This theory has been extended in [34] to include NI systems with poles in the closed left half of the complex plane, except at the origin. Also, further extensions to NI systems theory include the study of NI controller synthesis [35], [36], connections between NI systems analysis and $\mu$-analysis [37], and conditions for robust stability analysis of mixed NI and bounded-real classes of uncertainties [38]. Furthermore, the concept of lossless NI transfer functions is introduced in [39], an algebraic approach to the realization of a lossless NI behavior is presented in [40], and a spectral characterization of NI descriptor systems is discussed in [41]. The NI systems theory can be extended to nonlinear systems using the concept of counter-clockwise input-output dynamics as presented in [42]-[44]. In [44], a sufficient conditions under which a semilinear Duhem model is counter-clockwise is given, where the counter-clockwise input-output system is restricted to periodic input signals. positive feedback interconnection for SISO linear case is provided in [44].

Despite generalizations of the NI systems framework presented in [34], an important class of systems, that cannot be captured by the existing NI systems framework, corresponds to flexible systems with free body motion. These systems arise in areas such as rotating flexible spacecraft [45], rotary cranes [46], robotics and flexible link manipulators [27], [29], [47], and dual-stage hard disk drives [48]-[51]. Flexible structures with free body motion lead to dynamical models including poles at the origin, which is not covered in earlier work on NI systems theory. In particular, the stability condition (1) is not well defined in the case of flexible structures with free body motion which results in poles at the origin, since in this case, the plant DC gain $G(0)$ will be infinite. However, control systems involving flexible structures with free body motion arising in these important application areas still suffer from the stability and performance issues mentioned above. Thus we are motivated to extend the NI robust stability theory developed in [14], [16], [34] so that it can be applied to control systems involving highly resonant flexible structures with free body motion.

Fig. 2 shows a block diagram of a system which includes a flexible structure with free body motion that arises in a problem of disk-drive control; see [52]. Here, a voice coil 
motor (VCM) is used to actuate the arm of the reader head. The free body motion of the reader head leads to a transfer function from the input $V_{i n}$ to the VCM output $V_{\text {out }}$ which has poles at the origin. Furthermore, the overall system satisfies the NI frequency response property and includes poles at the origin. However, the NI stability results presented in [14], [16], [34] do not allow for poles at the origin and cannot be applied to control systems such as this disk-drive control system.

In this paper, we present a new generalized definition of NI systems which allows for flexible structures with colocated force actuators and position sensors and with free body motion. This definition extends the previous definitions of NI systems presented in [14], [16], [34] to allow for up to two poles at the origin. We also derive new generalized stability conditions for positive-feedback control systems involving an NI plant and an SNI controller.

As in [14], [16], [34], the stability conditions presented in this paper, are given purely in terms of properties of the plant and controller transfer function matrices, although the proofs rely on state space techniques. Furthermore, the stability conditions given are independent of the plant and controller system order and can be stated without using the fact that the plant and the controller transfer function matrices are rational. However, the proofs given in this paper only apply to the rational case.

Preliminary conference versions of the stability results presented in this paper were presented in [53], [54]. However, in this paper, much more general versions of these stability results are presented in Theorems 1 - 4 and Corollaries 1,2, which allow for the existence of free body motion in some but not all input-output channels. This is important since multivariable control systems involving flexible structures with free body motion usually include free body motion in some but not all input-output channels. Also, this paper includes a case study involving the control of a flexible robotic arm, which has not been considered in the previous conference versions of the paper.

This paper is further organized as follows: Section II recalls the existing definition for NI systems and outlines the notation that will be used in the rest of the paper. Section III introduces the new generalized definition for NI systems, which allows for systems with free body dynamics. Also in this section, we present the main stability results in Theorems $1-4$ and Corollaries 1-2. Section IV presents a case study, which involves a flexible robotic arm, as an application of the NI theory presented in this paper. The paper is concluded with a summary and remarks on future work in Section V. All proofs of the presented theorems, lemmas and corollaries are given in the Appendix.

\section{Preliminaries And Notation}

In this section, we recall the existing definitions of NI and SNI systems as given in [34] for systems with poles in the closed left half of the complex plane, except at the origin. We will use this existing definition of SNI (first introduced in [16]) systems but in the next section we will present our new definition of generalized NI systems. We also define notation used to describe positive feedback interconnections and internal stability, which will be used to present the main results in this paper.

Consider the following LTI system,

$$
\begin{aligned}
& \dot{x}(t)=A x(t)+B u(t), \\
& y(t)=C x(t)+D u(t),
\end{aligned}
$$

where $A \in \mathbb{R}^{n \times n}, B \in \mathbb{R}^{n \times m}, C \in \mathbb{R}^{m \times n}, D \in \mathbb{R}^{m \times m}$, and with the square transfer function matrix $G(s)=C(s I-$ $A)^{-1} B+D$. The transfer function matrix $G(s)$ is said to be strictly proper if $G(\infty)=D=0$. We will use the notation $\left[\begin{array}{l|l}A & B \\ \hline C & D\end{array}\right]$ to denote the state space realization (2), (3).

The existing definition of NI systems states that a square transfer function matrix $G(s)$ is NI if the following conditions are satisfied [34]:

1. $G(s)$ has no pole at the origin and in $\operatorname{Re}[s]>0$.

2. The corresponding frequency response $G(j \omega)$ is such that

$$
j\left(G(j \omega)-G(j \omega)^{*}\right) \geq 0,
$$

for all $\omega>0$ where $j \omega$ is not a pole of $G(s)$.

3. If $j \omega_{0}$ with $\omega_{0}>0$ is a pole of $G(s)$, it is at most a simple pole and the residue matrix $K_{0}=\lim _{j \rightarrow j \omega_{0}}(s-$ $\left.j \omega_{0}\right) s G(s)$ is positive semidefinite Hermitian.

Definition 1: [34] A square transfer function matrix $G(s)$ is SNI if the following conditions are satisfied:

1) $G(s)$ has no pole in $\operatorname{Re}[s] \geq 0$.

2) For all $\omega>0, j\left(G(j \omega)-G(j \omega)^{*}\right)>0$.

Now, consider a positive feedback interconnection between an NI system with transfer function matrix $G(s)$ and an SNI system with transfer function matrix $\bar{G}(s)$ as shown in Fig. 1. Also, suppose that the transfer function matrix $G(s)$ has a minimal state space realization $\left[\begin{array}{c|c}A & B \\ \hline C & D\end{array}\right]$, and $\bar{G}(s)$ has a
minimal state space realization $\left[\begin{array}{l|l}\bar{A} & \bar{B} \\ \hline C & D\end{array}\right]$. Furthermore, it is assumed that the matrix $I-D \bar{D}$ is nonsingular. Then the closed system has a system matrix given by

$$
\breve{A}=\left[\begin{array}{cc}
A+B \bar{D}(I-D \bar{D})^{-1} C & B \bar{C}+B \bar{D}(I-D \bar{D})^{-1} D \bar{C} \\
\bar{B}(I-D \bar{D})^{-1} C & \bar{A}+\bar{B}(I-D \bar{D})^{-1} D \bar{C}
\end{array}\right] .
$$

Moreover, the positive feedback interconnection between $G(s)$ and $\bar{G}(s)$ as shown in Fig. 1 and denoted $[G(s), \bar{G}(s)]$ is said to be internally stable if the closed-loop system matrix $\breve{A}$ in (4) is Hurwitz; e.g., see [55].

\section{MAIN RESUltS}

The main contribution of this paper is a generalization of the framework for NI systems presented in [34]. We introduce a new definition of NI systems that will allow for systems with free body dynamics. This generalized definition will be used in a new set of stability conditions that will allow for NI systems with free body motion to be included into the framework of 
NI systems theory. Henceforth, when a system is said to be NI, we will mean NI as defined below, not NI as defined in earlier papers.

Definition 2: A square transfer function matrix $G(s)$ is NI if the following conditions are satisfied:

1) $G(s)$ has no pole in $R e[s]>0$.

2) For all $\omega>0$ such that $j \omega$ is not a pole of $G(s)$, $j\left(G(j \omega)-G(j \omega)^{*}\right) \geq 0$.

3) If $s=j \omega_{0}$ with $\omega_{0}>0$ is a pole of $G(s)$, then it is a simple pole and the residue matrix $K=\lim _{s \rightarrow j \omega_{0}}(s-$ $\left.j \omega_{0}\right) j G(s)$ is Hermitian and positive semidefinite.

4) If $s=0$ is a pole of $G(s)$, then $\lim _{s \rightarrow 0} s^{k} G(s)=0$ for all $k \geq 3$ and $\lim _{s \longrightarrow 0} s^{2} G(s)$ is Hermitian and positive semidefinite.

Here, $G(j \omega)$ is the frequency response corresponding to the transfer function $G(s)$. Unlike the NI definition presented in [34], Definition 2 allows for poles at the origin. In this case, we cannot use the existing stability results presented in [14], [34], [35], because the stability condition in (1) is not defined. The inclusion of poles at the origin extends the NI systems theory to include flexible systems with free body dynamics. In order to derive a set of stability conditions that allow for NI systems with free body motion, we define the following constant matrices for a given $m \times m$ NI transfer function matrix $G(s)$ :

$$
\begin{aligned}
G_{2} & =\lim _{s \rightarrow 0} s^{2} G(s), \\
G_{1} & =\lim _{s \rightarrow 0} s\left(G(s)-\frac{G_{2}}{s^{2}}\right), \\
G_{0} & =\lim _{s \rightarrow 0}\left(G(s)-\frac{G_{2}}{s^{2}}-\frac{G_{1}}{s}\right) .
\end{aligned}
$$

These matrices are the first three coefficients in the Laurent series expansion of the transfer function $G(s)$. These matrices carry information about properties of the free body motion of the system under consideration and will be used in stability conditions for the positive feedback interconnection of NI and SNI systems. Note that the DC gain condition (1) cannot be defined for an NI system with transfer function matrix $G(s)$ unless $G_{2}=G_{1}=0$, which reduces to the case where the dynamical system has no free body motion. From Condition 4) in Definition 2, the matrix $G_{2}$ is required to be Hermitian and positive semidefinite. Hence, it follows (e.g., see [56]) that if $G_{2} \neq 0$, it can be decomposed in the form

$$
G_{2}=J J^{T}
$$

where $J$ is a full column rank matrix.

We now present conditions for the stability of a positive feedback control system involving an NI plant with free body motion. These conditions are stated using the quantities defined in (5). First, we define the $2 m \times 2 m$ Hankel matrix $\Gamma$ as

$$
\Gamma=\left[\begin{array}{cc}
G_{1} & G_{2} \\
G_{2} & 0
\end{array}\right]
$$

Suppose that $\Gamma \neq 0$. Using the singular value decomposition (SVD), we can decompose the Hankel matrix $\Gamma$ as

$$
\begin{aligned}
\Gamma & =\left[\begin{array}{ll}
H_{1} & H_{2}
\end{array}\right]\left[\begin{array}{ll}
S & 0 \\
0 & 0
\end{array}\right]\left[\begin{array}{c}
V_{1}^{T} \\
V_{2}^{T}
\end{array}\right] \\
& =H_{1} S V_{1}^{T}=U V_{1}^{T}=\left[\begin{array}{l}
U_{1} \\
U_{2}
\end{array}\right] V_{1}^{T},
\end{aligned}
$$

where $\left[\begin{array}{ll}H_{1} & H_{2}\end{array}\right],\left[\begin{array}{c}V_{1}^{T} \\ V_{2}^{T}\end{array}\right]$ are unitary matrices, $S>0, U=$ $H_{1} S \in \mathbb{R}^{2 m \times \tilde{n}}, U_{1} \in \mathbb{R}^{m \times \tilde{n}}, U_{2} \in \mathbb{R}^{m \times \tilde{n}}$ and the matrices $U$ and $V_{1}$ each have orthogonal columns. Furthermore, we can decompose the $\tilde{n} \times \tilde{n}$ matrix $U_{1}^{T} U_{2}$ using the SVD as

$$
U_{1}^{T} U_{2}=\hat{U} \hat{S} \hat{V}^{T}=\hat{U}\left[\begin{array}{cc}
S_{1} & 0 \\
0 & 0
\end{array}\right]\left[\begin{array}{l}
\hat{V}_{1}^{T} \\
\hat{V}_{2}^{T}
\end{array}\right],
$$

where $\hat{U} \in \mathbb{R}^{\tilde{n} \times \tilde{n}}$ and $\hat{V} \in \mathbb{R}^{\tilde{n} \times \tilde{n}}$ are orthogonal matrices, $\hat{V}_{2} \in \mathbb{R}^{\tilde{n} \times \check{n}}$ and $S_{1}>0$.

We now introduce some notation which will be used throughout the paper. Given matrices $X \in \mathbb{R}^{m \times m}$ and $Y \in \mathbb{R}^{m \times \check{n}}$ such that $\operatorname{det}\left(Y^{T} X Y\right) \neq 0$, then the matrix valued function $\mathcal{P}(X, Y)$ is defined by

$$
\mathcal{P}(X, Y) \triangleq X-X Y\left(Y^{T} X Y\right)^{-1} Y^{T} X .
$$

Using this notation, we define the matrix

$$
N_{f}=\mathcal{P}(\bar{G}(0), F),
$$

where the $m \times \check{n}$ matrix $F$ is given by

$$
F=U_{1} \hat{V}_{2}
$$

and we will assume that $\operatorname{det}\left(F^{T} \bar{G}(0) F\right) \neq 0$.

We will use the following condition in the theorem which follows:

$$
F^{T} \bar{G}(0) F<0 .
$$

Also, for the case in which $N_{f}$ is positive semidefinite, we will use the condition

$$
I-N_{f}^{\frac{1}{2}} G_{0} N_{f}^{\frac{1}{2}}-N_{f}^{\frac{1}{2}} G_{1} J\left(J^{T} J\right)^{-2} J^{T} G_{1}^{T} N_{f}^{\frac{1}{2}}>0 .
$$

Moreover, for the case in which $N_{f}$ is negative semidefinite, we will use the condition

$$
\operatorname{det}\left(I+\tilde{N}_{f} G_{0} \tilde{N}_{f}+\tilde{N}_{f} G_{1} J\left(J^{T} J\right)^{-2} J^{T} G_{1}^{T} \tilde{N}_{f}\right) \neq 0 .
$$

Here, $\tilde{N}_{f}=\left(-N_{f}\right)^{\frac{1}{2}}$ and matrices $G_{1}, G_{0}, J, N_{f}$ and $F$ are defined in (5), (6), (11), and (12) respectively. Also, (.) $)^{\frac{1}{2}}$ denotes the square root of a positive semidefinite matrix.

The following theorem is our first main stability result for the case in which $G_{2} \neq 0$. That is, the system has double poles at the origin.

Theorem 1: Suppose that the square transfer function matrix $G(s)$ is strictly proper and NI with $G_{2} \neq 0$, and the transfer function matrix $\bar{G}(s)$ is SNI. Also, suppose that the matrix $F^{T} \bar{G}(0) F$ is non-singular. If $N_{f}$ is positive semidefinite, then the closed-loop positive-feedback interconnection between $G(s)$ and $\bar{G}(s)$ as shown in Fig. 1 is internally stable 
if and only if conditions (13) and (14) are satisfied. Furthermore, if $N_{f}$ is negative semidefinite, then the closed-loop positive-feedback interconnection between $G(s)$ and $\bar{G}(s)$ is internally stable if and only if conditions (13) and (15) are satisfied.

The proof of this and subsequent theorems and corollaries are presented in Appendix B.

We now present a corollary to this theorem which considers the special case in which none of the free body modes of the plant have frictional force present; i.e., $G_{1}=0$. In order to present this corollary, we define the matrix $N_{2}$ as follows:

$$
N_{2}=\mathcal{P}(\bar{G}(0), J),
$$

where we assume that the matrix $J^{T} \bar{G}(0) J$ is non-singular.

We will use the following condition in the next corollary, which corresponds to condition (13) in Theorem 1:

$$
J^{T} \bar{G}(0) J<0 .
$$

Also, for the case in which $N_{2}$ is positive semidefinite, we will use the following condition which corresponds to condition (14) in Theorem 1:

$$
I-N_{2}^{\frac{1}{2}} G_{0} N_{2}^{\frac{1}{2}}>0
$$

Moreover, for the case in which $N_{2}$ is negative semidefinite, we will use the following condition which corresponds to condition (15) in Theorem 1:

$$
\operatorname{det}\left(I+\tilde{N}_{2} G_{0} \tilde{N}_{2}\right) \neq 0,
$$

where $\tilde{N}_{2}=\left(-N_{2}\right)^{\frac{1}{2}}$.

Corollary 1: Suppose that the transfer function matrix $\bar{G}(s)$ is SNI and the strictly proper transfer function matrix $G(s)$ is NI with $G_{1}=0$ and $G_{2} \neq 0$. Also, suppose that the matrix $J^{T} \bar{G}(0) J$ is non-singular. If $N_{2}$ is positive semidefinite, then the closed-loop positive-feedback interconnection between $G(s)$ and $\bar{G}(s)$ is internally stable if and only if conditions (17) and (18) are satisfied. Furthermore, if $N_{2}$ is negative semidefinite, then the closed-loop positive-feedback interconnection between $G(s)$ and $\bar{G}(s)$ is internally stable if and only if conditions (17) and (19) are satisfied.

The following theorem imposes some extra conditions on the matrix $G_{2}$ which enables us to relax the sign definiteness condition on the matrix $N_{2}$. This then leads to a simplified stability condition.

Theorem 2: Suppose that the transfer function matrix $\bar{G}(s)$ is SNI and the strictly proper transfer function matrix $G(s)$ is NI with $G_{1}=0$ and $G_{2} \neq 0$. Also, suppose that $\mathcal{N}\left(G_{2}\right) \subseteq \mathcal{N}\left(G_{0}^{T}\right)$, where $\mathcal{N}(\cdot)$ denotes the null space of a matrix. Then the closed-loop positive-feedback interconnection between $G(s)$ and $\bar{G}(s)$ is internally stable if and only if condition (17) is satisfied.

In Theorem 3, Theorem 4 and Corollary 2, we consider cases which correspond to free body motion with frictional force present. As in Theorem 1, these cases allow for fact that the free body motion may not be present in all input-output channels.

In order to present Theorem 3 and Theorem 4, suppose that $G_{1} \neq 0$ and $G_{2}=0$. This corresponds to the case when the system has a single pole at the origin. Then we consider the following SVD decomposition of the matrix $G_{1}$ defined in (5):

$$
G_{1}=\left[\begin{array}{ll}
\tilde{F}_{1} & \tilde{F}_{2}
\end{array}\right]\left[\begin{array}{cc}
S_{2} & 0 \\
0 & 0
\end{array}\right]\left[\begin{array}{l}
V_{1}^{T} \\
V_{2}^{T}
\end{array}\right]=F_{1} V_{1}^{T},
$$

where $S_{2}>0$, and the matrices $F_{1}=\tilde{F}_{1} S_{2}$ and $V_{1}$ each have orthogonal columns. Also, we define the matrix $N_{1}$ as follows:

$$
N_{1}=\mathcal{P}\left(\bar{G}(0), F_{1}\right)
$$

where the matrix $F_{1}^{T} \bar{G}(0) F_{1}$ is assumed to be non-singular.

We will use the following condition in Theorem 3 and Corollary 2 which corresponds to condition (13) in Theorem 1 :

$$
F_{1}^{T} \bar{G}(0) F_{1}<0
$$

For the case in which $N_{1}$ is positive semidefinite, we also will use the following condition which corresponds to condition (14) in Theorem 1:

$$
I-N_{1}^{\frac{1}{2}} G_{0} N_{1}^{\frac{1}{2}}>0 .
$$

Moreover, for the case in which $N_{1}$ is negative semidefinite, we will use the following condition which corresponds to condition (15) in Theorem 1:

$$
\operatorname{det}\left(I+\tilde{N}_{1} G_{0} \tilde{N}_{1}\right) \neq 0,
$$

where $\tilde{N}_{1}=\left(-N_{1}\right)^{\frac{1}{2}}$.

Theorem 3: Suppose that the transfer function matrix $\bar{G}(s)$ is SNI and the strictly proper transfer function matrix $G(s)$ is NI with $G_{2}=0$ and $G_{1} \neq 0$. Also, suppose that the matrix $F_{1}^{T} \bar{G}(0) F_{1}$ non-singular. If $N_{1}$ is positive semidefinite, then the closed-loop positive-feedback interconnection between $G(s)$ and $\bar{G}(s)$ is internally stable if and only if conditions (22) and (23) are satisfied. Furthermore, if $N_{1}$ is negative semidefinite, then the closed-loop positive-feedback interconnection between $G(s)$ and $\bar{G}(s)$ is internally stable if and only if conditions (22) and (24) are satisfied.

The following theorem imposes some extra conditions on the matrix $G_{1}$ which enables us to relax the sign definiteness condition on the matrix $N_{1}$. This then leads to a simplified stability condition.

Theorem 4: Suppose that the transfer function matrix $\bar{G}(s)$ is SNI and the strictly proper transfer function matrix $G(s)$ is NI with $G_{2}=0$ and $G_{1} \neq 0$. Also, suppose that $\mathcal{N}\left(G_{1}^{T}\right) \subseteq \mathcal{N}\left(G_{0}^{T}\right)$. Then the closed-loop positive-feedback interconnection between $G(s)$ and $\bar{G}(s)$ is internally stable if and only if condition (22) is satisfied.

The following corollary presents an important special case of Theorem 2 and 4.

Corollary 2: Suppose that the transfer function matrix $\bar{G}(s)$ is SNI and the strictly proper transfer function matrix $G(s)$ is NI with either $G_{2}=0$ and $G_{1}$ invertible or $G_{1}=0$ and $G_{2}>0$. Then, the closed-loop positive-feedback interconnection between $G(s)$ and $\bar{G}(s)$ is internally stable if and only if $\bar{G}(0)<0$.

Remark 1: The case where $G_{2}=0$ and $G_{1}=0$ cor- 
responds to the existing stability results presented in [14], [34], [35]. In this case, the stability condition reduces to $\lambda_{\max }(\bar{G}(0) G(0))<1$. This condition can be obtained from (23) using the fact $N_{1}=\bar{G}(0)$ in this case. Also, we require the assumption $\bar{G}(0)>0$. Hence,

$$
\begin{aligned}
& I-N_{1}^{\frac{1}{2}} G_{0} N_{1}^{\frac{1}{2}}>0, \\
\Leftrightarrow & N_{1}^{-1}-G_{0}>0, \\
\Leftrightarrow & \lambda_{\max }\left(\bar{G}(0) G_{0}\right)<1 .
\end{aligned}
$$

Note that using a similar argument to the proof of Theorem 3 , we can obtain a similar result under the assumption that $\bar{G}(0)<0$.

\section{Case Study: Control of Flexible robotic arm}

In this section, we present an application of the stability results presented in this paper to the control of a flexible robotic arm system. The robotic arm is pinned to a motor at one end. For the purposes of modeling the flexible robotic arm, we use an equivalent slewing beam model as depicted in Fig. 3; see [57]. The motor allows the robotic arm to traverse

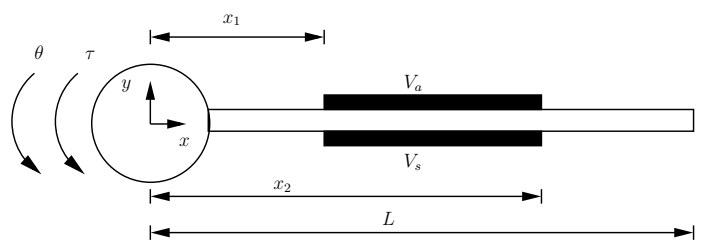

Fig. 3. Schematic diagram of the slewing beam equivalent to the robotic arm.

in the vertical plane. Two piezoelectric patches are attached to the arm on either side. Here, one piezoelectric patch acts as an actuator while the other is a sensor. The robotic arm system has two inputs and two outputs: the inputs are the voltage $V_{a}$ applied to the piezoelectric actuator and the torque $\tau$ applied by the motor, whereas the outputs are the voltage $V_{s}$ produced by the piezoelectric sensor and the motor hub angle $\theta$. The fact that this system involves colocated "force" actuators and "position" sensors indicates that the system will be NI; e.g., see [14].

\section{A. Mathematical model for the robotic arm}

The beam in Fig. 3 is modeled using the Bernoulli-Euler equations of motion for a beam with actuating and sensing piezoelectric elements as in [57]:

$$
\frac{\partial^{2}}{\partial x^{2}}\left[E I \frac{\partial^{2}}{\partial y^{2}} y(x, t)-C_{a} V_{a}(x, t)\right]+\rho A \frac{\partial^{2}}{\partial t^{2}} y(x, t)=0 .
$$

Here, $E$ is Young's modulus and $I$ is the second moment of inertia of the beam, $\rho$ is the density of the beam, and $A$ is the area of the composite beam. If the thickness of the piezoelectric films are comparable to the thickness of the beam, then the products $E I$ and $\rho A$ would be different in the laminated and non-laminated areas of the beam. However, since piezoelectric films used in practical applications are often thin compared to the thickness of the beam, these differences will be neglected. Assuming that the products $E I$ and $\rho A$ are uniform over the length of the beam simplifies the modeling procedure.

Now we consider various boundary conditions in modeling the beam. These are given as

$$
\begin{aligned}
& y(0, t)=0, \\
& E I \frac{\partial^{2}}{\partial x^{2}} y(0, t)-I_{h} \frac{\partial^{3}}{\partial t^{2} \partial x} y(0, t)+\tau(t)=0, \\
& E I \frac{\partial^{2}}{\partial x^{2}} y(L, t)+I_{t} \frac{\partial^{3}}{\partial t^{2} \partial x} y(L, t)=0, \\
& E I \frac{\partial^{3}}{\partial y^{3}} y(L, t)-M_{t} \frac{\partial^{3}}{\partial t^{2} \partial x} y(L, t)=0 .
\end{aligned}
$$

Here, $M_{t}$ and $I_{t}$ are the mass and inertia of the tip, which will be neglected in this paper.Also, (26) represents the inability of the motor joint to undergo transverse motion. As in [57], the time domain beam equation (25) with boundary conditions (26)-(29) can be transformed into an equivalent Laplace domain representation as

$$
Y^{\prime \prime \prime \prime}(x, s)-\beta^{4} Y(x, s)=\frac{C_{a} V_{a}^{\prime \prime}(x, s)}{E I}
$$

with boundary conditions

$$
\begin{aligned}
& Y(0, s)=0, \\
& E I Y^{\prime \prime}(0, s)-I_{h} s^{2} Y^{\prime}(0, s)+\tau(s)=0, \\
& E I Y^{\prime \prime}(L, s)+I_{t} s^{2} Y^{\prime}(L, s)=0, \\
& E I Y^{\prime \prime \prime}(L, s)-M_{t} s^{2} Y(L, s)=0,
\end{aligned}
$$

where the primes indicate spatial derivatives and

$$
\beta^{4}(s)=-\frac{\rho A s^{2}}{E I} .
$$

Note that (30) is the Laplace domain equivalent of the Bernoulli-Euler beam equation with $V_{a}^{\prime \prime}(\cdot)$ as a forcing input. Together, (30)-(34) represent a set of linear ordinary differential equations with mixed boundary conditions: two at $x=0$ and two at $x=L$. A state space representation for the system can be formed from equations (30)-(34) as in [57]:

$$
\begin{aligned}
{\left[\begin{array}{c}
Y^{\prime}(x, s) \\
Y^{\prime \prime}(x, s) \\
Y^{\prime \prime \prime}(x, s) \\
Y^{\prime \prime \prime \prime}(x, s)
\end{array}\right]=} & {\left[\begin{array}{llll}
0 & 1 & 0 & 0 \\
0 & 0 & 1 & 0 \\
0 & 0 & 0 & 1 \\
\beta^{4} & 0 & 0 & 0
\end{array}\right]\left[\begin{array}{c}
Y(x, s) \\
Y^{\prime}(x, s) \\
Y^{\prime \prime}(x, s) \\
Y^{\prime \prime \prime}(x, s)
\end{array}\right] } \\
& +\left[\begin{array}{l}
0 \\
0 \\
0 \\
1
\end{array}\right] \frac{C_{a} V_{a}(s)}{E I} \sum_{i=1}^{2} \delta\left(x-x_{i}\right)(-1)^{i+1}
\end{aligned}
$$

where $\delta(\cdot)$ represents the Dirac delta function. The equation (36) can be written in the general form

$$
Z^{\prime}(x, s)=\bar{A} Z(x, s)+\bar{B} U(x, s),
$$

the solution to which is given by

$$
\begin{aligned}
& Z(x, s) \\
& =e^{\bar{A} x} Z(0, s)+\left[\bar{A} e^{\bar{A}\left(x-x_{1}\right)} \bar{B}-\bar{A} e^{\bar{A}\left(x-x_{2}\right)} \bar{B}\right] \frac{C_{a} V_{a}(s)}{E I} .
\end{aligned}
$$

Once the boundary conditions $Z(0, s)$ and $Z(L, s)$ are known, (38) will depend upon three conditions for $x$, namely, $0 \leq x \leq$ $x_{1}, x_{1} \leq x \leq x_{2}$, and $x_{2} \leq x \leq L$. For further details see 
[57].

\section{B. Infinite Dimensional Transfer function Model}

Here, we present the input-output relationship between the two inputs $V_{a}$ and $\tau$, and the corresponding collocated outputs $V_{s}$ and $\theta$ in the form of the transfer function matrix,

$$
\left[\begin{array}{c}
\theta(s) \\
V_{s}(s)
\end{array}\right]=G(s)\left[\begin{array}{c}
\tau(s) \\
V_{a}(s)
\end{array}\right]
$$

where $G(s)=\left[\begin{array}{cc}G_{\tau, \theta}(s) & G_{V_{a}, \theta}(s) \\ G_{\tau, V_{s}}(s) & G_{V_{a}, V_{s}}(s)\end{array}\right]$ and each of the elements of this transfer function matrix is an infinite dimensional transfer function defined in terms of transcendental functions of $\beta$. Indeed, each of the four transfer functions in (39) can be written as a ratio of numerator and denominator functions computed as

$$
\begin{aligned}
& G_{\tau, \theta}(s)=\frac{N_{\tau, \theta}(s)}{D(s)}=\left.\frac{Y^{\prime}(0, s)}{T}\right|_{V_{a}(s)=0}, \\
& G_{V_{a}, \theta}(s)=\frac{N_{V_{a}, \theta}(s)}{D(s)}=\left.\frac{Y^{\prime}(0, s)}{V_{a}}\right|_{\tau(s)=0}, \\
& G_{\tau, V_{s}}(s)=\frac{N_{\tau, V_{s}}(s)}{D(s)}=\left.\frac{C_{s}\left(Y^{\prime}\left(x_{2}, s\right)-Y^{\prime}\left(x_{1}, s\right)\right)}{\tau(s)}\right|_{V_{a}(s)=0}, \\
& G_{V_{a}, V_{s}}(s)=\frac{N_{V_{a}, V_{s}}(s)}{D(s)}=\left.\frac{C_{s}\left(Y^{\prime}\left(x_{2}, s\right)-Y^{\prime}\left(x_{1}, s\right)\right)}{V_{a}(s)}\right|_{T(s)=0} .
\end{aligned}
$$

Here,

$$
\begin{aligned}
D(s)= & 4 \beta E I(\rho A(\cos (\beta l) \sinh (\beta l)-\cosh (\beta l) \sin (\beta l))) \\
& \left.-4 \beta^{4} E I I_{h}(1+\cos (\beta l)) \cosh (\beta l)\right),
\end{aligned}
$$

where $I_{h}$ is the hub inertia. Also, the functions $N_{\tau, \theta}(s), N_{V_{a}, \theta}, N_{\tau, V_{s}}(s), N_{V_{a}, V_{s}}(s)$ are given by very complicated expressions which can be found in equations (26)-(28) in [57].

We now compute the transfer functions in (40)-(43) for the case where the piezoelectric actuators and sensors span the entire length of the beam. This corresponds to the substitutions: $x_{1}=0$ and $x_{2}=L$. The resulting transfer functions have been verified in [58] for an experimented robotic arm system.

Despite the fact that we have not defined the NI property for infinite dimensional transfer functions, we will provide some calculations which indicate that the infinite dimensional transfer function matrix $G(s)$ defined in (39)-(43) satisfies the NI conditions given in Definition 2. Since the infinite dimensional transfer function matrix $G(s)$ is actually a transcendental function of $\beta(s), G(s)=\tilde{G}(\beta(s))$, then Condition 2 ) in Definition 2 is equivalent to the condition

$$
j\left(\tilde{G}(\beta(j \omega))-\tilde{G}(\beta(j \omega))^{*}\right) \geq 0
$$

for all $\omega \geq 0$ where $\beta(s)$ is given by (35). Indeed, it is straightforward to verify from the formulas for the transfer function matrix (39)-(43) that $j\left(\tilde{G}(\beta(j \omega))-\tilde{G}(\beta(j \omega))^{*}\right)=0$ for all $\omega \geq 0$. Also, the function $D(s)$ given in (44) has an infinite numbers of roots. However, we can check Condition 3 ) in Definition 2 for a finite number of these roots on the imaginary axis. To do so, we have calculated the first eleven $j \omega$-axis roots of $D(s)$ numerically. At each of these roots $s=j \omega_{0}$, the corresponding residue matrix $K\left(j \omega_{0}\right)=$ $\lim _{\rightarrow j \omega_{0}}\left(s-j \omega_{0}\right) j G(s)$ is calculated using L'Hopital's rule as follows:

$$
\begin{aligned}
K\left(j \omega_{0}\right) & =\lim _{s \longrightarrow j \omega_{0}}\left(s-j \omega_{0}\right) j G(s) \\
& =\lim _{s \longrightarrow j \omega_{0}}\left(s-j \omega_{0}\right) j\left[\begin{array}{ll}
\frac{N_{\tau, \theta}(s)}{D(s)} & \frac{N_{V_{a}, \theta}(s)}{D(s)} \\
\frac{N_{\tau, V_{s}}(s)}{D(s)} & \frac{N_{V_{a}, V_{s}}(s)}{D(s)}
\end{array}\right] \\
& =j\left[\begin{array}{ll}
\frac{N_{\tau, \theta}\left(j \omega_{0}\right)}{D^{\prime}\left(j \omega_{0}\right)} & \frac{N_{V_{a}, \theta}\left(j \omega_{0}\right)}{D^{\prime}\left(j \omega_{0}\right)} \\
\frac{N_{\tau, V_{s}}\left(j \omega_{0}\right)}{D^{\prime}\left(j \omega_{0}\right)} & \frac{N_{V_{a}, V_{s}}\left(j \omega_{0}\right)}{D^{\prime}\left(j \omega_{0}\right)}
\end{array}\right],
\end{aligned}
$$

where $D^{\prime}(j \omega)$ denotes the first derivative of $D(j \omega)$ with respect to $\omega$.

In this case study, the parameter values for the robotic arm are taken from [58]. These parameter values are shown in the Table I.

TABLE I

ROBOTIC ARM PARAMETER VALUES.

\begin{tabular}{|l|c|c|}
\hline \hline Parameter & Value & Unit \\
\hline Hub inertia, $I_{h}$ & 0.0348 & $N-m-s^{2}$ \\
\hline Beam length, $l$ & 2 & $m$ \\
\hline Volumetric mass density, $\rho$ & 2712.6 & $\mathrm{Kg} / \mathrm{m}^{2}$ \\
\hline Cross sectional area, $A$ & $483.87 \times 10^{-6}$ & $\mathrm{~m}^{2}$ \\
\hline Young's Modulus, $E$ & $69.0 \times 10^{9}$ & $\mathrm{~N} / \mathrm{m}^{2}$ \\
\hline Area moment of inertia, $I$ & $1.63 \times 10^{-9}$ & $\mathrm{~m}^{4}$ \\
\hline Coupling Coefficient $k_{31}$ & -0.340 & - \\
\hline Capacitance, $C$ & 68.35 & $\mu \mathrm{F} / \mathrm{m}^{2}$ \\
\hline Thickness $t_{s}$ & $3.05 \times 10^{-4}$ & $\mathrm{~m}$ \\
\hline \hline
\end{tabular}

Table II shows the calculated roots of $D(s)$ and the minimum eigenvalue of the corresponding residue matrix given in (46). Also, the matrix $G_{2}=\lim _{s \rightarrow 0} s^{2} G(s)$ is found to be $\left[\begin{array}{cc}0.14 & 0 \\ 0 & 0\end{array}\right]$ which is positive semidefinite. These results show

TABLE II

THE MINIMUM EIGENVALUES OF THE RESIDUE MATRIX CORRESPONDING TO THE FIRST TEN RESONANT MODES OF THE INFINITE DIMENSIONAL PLANT TRANSFER FUNCTION MATRIX.

\begin{tabular}{|c|c|c|}
\hline \hline $\mathrm{n}$ & Root $s=j \omega(\mathrm{rad} / \mathrm{s})$ & Minimum eigenvalue \\
\hline 0 & 0 & 0 \\
\hline 1 & 3.395326441 & 0.1434 \\
\hline 2 & 9.501801884 & 0.2553 \\
\hline 3 & 17.08210071 & 0.1320 \\
\hline 4 & 29.32863976 & 0.0361 \\
\hline 5 & 47.01240951 & 0.0142 \\
\hline 6 & 96.84550724 & 0.0049 \\
\hline 7 & 128.7332003 & 0.0034 \\
\hline 8 & 165.2195349 & 0.0025 \\
\hline 9 & 206.2898971 & 0.0019 \\
\hline 10 & 251.9420283 & 0.0015 \\
\hline
\end{tabular}

that the infinite dimensional transfer function matrix $G(s)$ satisfies the conditions of Definition 2, at least for the first ten resonant modes.

Also in the Fig. 4, we plot the $\log$ of the minimum eigenvalue of the matrix $D^{\prime}(j \omega)^{2} K(j \omega)+\gamma D(j \omega)^{2}$ as a 


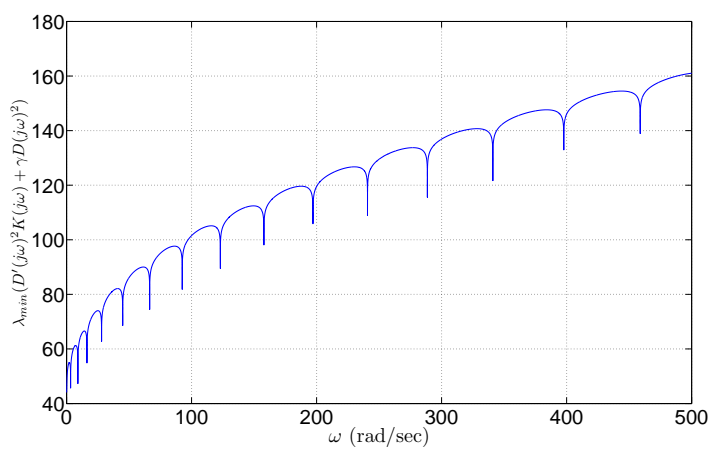

Fig. 4. Plot of the $\log$ of the minimum eigenvalue of the matrix $D^{\prime}(j \omega)^{2} K(j \omega)+\gamma D(j \omega)^{2}$ versus frequency $\omega$.

function of frequency $\omega$, where $\gamma$ is a positive constant. This plot also indicates that the residue matrix defined in (46) will be positive semidefinite at the system poles within the frequency range of interest.

\section{Approximate Finite-dimensional Transfer Function Matrix}

The transfer functions in (40)-(43) are irrational functions of $s$. We now approximate these transfer functions by rational functions in $s$ in order to design a suitable controller for the robotic arm system and to simulate its performance. Various methods such as the Maclaurin series expansion presented in [59], the Rayleigh-Ritz method [60], and the assumed modes method [60] are available in literature for the finite dimensional approximation of such an infinite dimensional model. Here, we adopt a partial fraction approach to obtain a finite dimensional approximation of $G(s)$. This method is similar to the assumed modes technique described in [60]. The finite dimensional model can be written as

$$
\begin{aligned}
G_{f}(s) & =\left[\begin{array}{cc}
G_{f \tau, \theta}(s) & G_{f V_{a}, \theta}(s) \\
G_{f \tau, V_{s}}(s) & G_{f V_{a}, V_{s}}(s)
\end{array}\right] \\
& =\left[\begin{array}{ll}
\frac{N_{\tau, \theta}(s)}{D(s)} & \frac{N_{V_{a}, \theta}(s)}{D(s)} \\
\frac{N_{\tau, V_{s}}(s)}{D(s)} & \frac{N_{V_{a}, V_{s}}(s)}{D(s)}
\end{array}\right] \\
& =\sum_{i=0}^{n} \frac{1}{k}\left[\begin{array}{ll}
\frac{a_{i}}{s^{2}+p_{i}^{2}} & \frac{b_{i}}{s^{2}+p_{i}^{2}} \\
\frac{c_{i}}{s^{2}+p_{i}^{2}} & \frac{d_{i}}{s^{2}+p_{i}^{2}}
\end{array}\right] .
\end{aligned}
$$

Here $D(s)$ in the infinite dimensional model $G(s)$ is approximated by

$$
D_{f}(s)=k \prod_{i=0}^{n}\left(s^{2}+p_{j}^{2}\right),
$$

where, $j p_{0} \ldots j p_{n}$ are the first $\mathrm{n} j \omega$-axis roots of $D(s)$. Also, the coefficient matrices $C_{i}=\left[\begin{array}{ll}a_{i} & b_{i} \\ c_{i} & d_{i}\end{array}\right]$, are computed using a partial fraction expansion method. That is,

$$
C_{i}=\frac{1}{k \prod_{j=0, j \neq i}^{n}\left(-p_{i}^{2}+p_{j}^{2}\right)}\left[\begin{array}{cc}
N_{\tau, \theta}\left(j p_{i}\right) & N_{V_{a}, \theta}\left(j p_{i}\right) \\
N_{\tau, V_{s}}\left(j p_{i}\right) & N_{V_{a}, V_{s}}\left(j p_{i}\right)
\end{array}\right] .
$$

The constant $k$ is chosen so that

$$
D\left(j \omega_{0}\right)=k \prod_{i=0}^{n}\left(-\omega_{0}^{2}+p_{i}^{2}\right)
$$

where $\omega_{0}$ is such that $j \omega_{0}$ is not a root of $D(s)$. We consider the first resonant mode; i.e., $n=1$ for the con- troller design. The corresponding coefficient matrices were computed and were found to be $C_{0}=\left[\begin{array}{cc}0.14 & 0 \\ 0 & 0\end{array}\right] ; C_{1}=$ $\left[\begin{array}{cc}3.0907 & 3.5573 \times 10^{-4} \\ 3.5573 \times 10^{-4} & 2.3500\end{array}\right] ;$ and $k=6.6667 \times 10^{-8}$. Also, the poles were computed to be $p_{0}=0, p_{1}=3.4$.

The finite dimensional model $G_{f}(s)$ in (47) is NI, since $j\left(G_{f}(j \omega)-G_{f}(j \omega)^{*}\right)=0$ for all $\omega \geq 0$, where $j \omega$ is not a pole for $G_{f}(s)$. This follows because in this example, $G_{f}(j \omega)$ is real and symmetric for all $\omega$ such that $j \omega$ is not a pole of $G_{f}(s)$. Also, the coefficient matrices $C_{0}, C_{1}$ are positive semidefinite which implies that Condition 3) in Definition 2 is satisfied. Moreover, $G_{2}=\lim _{s \rightarrow 0} s^{2} G(s)=C_{0} \geq 0$, which implies that Condition 4) in Definition 2 is satisfied.

\section{Controller design}

According to Theorem 1 if a plant is NI, any SNI controller which satisfies the conditions of Theorem 1 will stabilize the system. The fact that the robotic arm plant involves colocated "force" actuators and "position" sensors indicates that this plant should be NI. In particular, the finite dimensional approximation to the robotic arm model derived in Subsection IV-C was shown to be NI. We will now use a finite dimensional model of the form (47) to design a controller for the system. First, we compute the matrices $G_{2}, G_{1}$, and $G_{0}$ in (5), for the finite dimension approximate system where $n=1$ in (47) to obtain

$$
\begin{aligned}
G_{2} & =\left[\begin{array}{cc}
0.14 & 0 \\
0 & 0
\end{array}\right] \geqslant 0 ; \quad G_{1}=\left[\begin{array}{ll}
0 & 0 \\
0 & 0
\end{array}\right] ; \\
G_{0} & =\left[\begin{array}{cc}
0.41253083 & 0.0000319 \\
0.0000319 & 0.15672805
\end{array}\right] .
\end{aligned}
$$

This implies that we can use Corollary 1 to guarantee the stability of the positive feedback interconnection between the plant and an SNI controller.

In this case study, an integral resonant controller (IRC) is chosen to stabilize the system; e.g., see [14]. An IRC is a first order controller which takes the form

$$
\bar{G}(s)=(s I+\Gamma \Phi)^{-1} \Gamma-\Delta .
$$

This controller is SNI if $\Gamma>0, \Phi>0$ and $\Delta$ is a symmetric matrix [14]. Now, we chose the controller matrices $\Gamma>0, \Phi>$ 0 and $\Delta$ such that the conditions of Corollary 1 are satisfied. We choose the controller matrices as follows:

$$
\Gamma=\left[\begin{array}{ll}
35 & 15 \\
15 & 20
\end{array}\right] ; \Phi=\left[\begin{array}{ll}
0.745 & 0.521 \\
0.521 & 1.021
\end{array}\right] ; \Delta=\left[\begin{array}{cc}
4.2900 & 0 \\
0 & 2.22
\end{array}\right] \text {. }
$$

This leads to a controller DC gain matrix of $\bar{G}(0)=$ $\left[\begin{array}{ll}-2.2029 & -1.0650 \\ -1.0650 & -0.6971\end{array}\right]$. To check the stability conditions in Corollary 1, we first compute the matrix $J$ in (6) using $G_{2}$ in (51). This yields $J=\left[\begin{array}{c}0.3751 \\ 0\end{array}\right]$. Also, the matrix $N_{2}$ in (16) is calculated as $N_{2}=\left[\begin{array}{cc}0 & 0 \\ 0 & -0.182252\end{array}\right]$, which is negative semidefinite. Then we conclude $\operatorname{det}\left(I+\tilde{N}_{2} G_{0} \tilde{N}_{2}\right)=$ $\operatorname{det}\left[\begin{array}{cc}1.000000025 & 0.000000003 \\ 0.000000003 & 5.390603\end{array}\right] \neq 0$, where $\tilde{N}_{2}=\left(-N_{2}\right)^{1 / 2}$. 


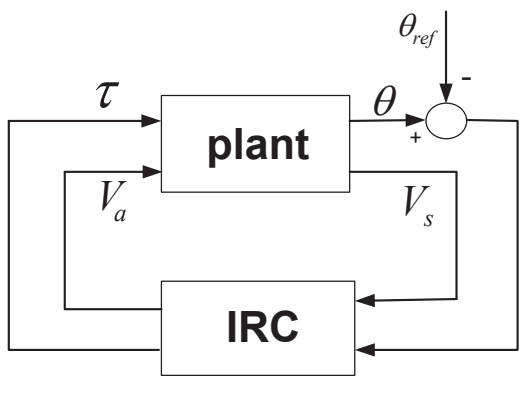

Fig. 5. Block diagram corresponding to a step change in the robotic arm reference position.

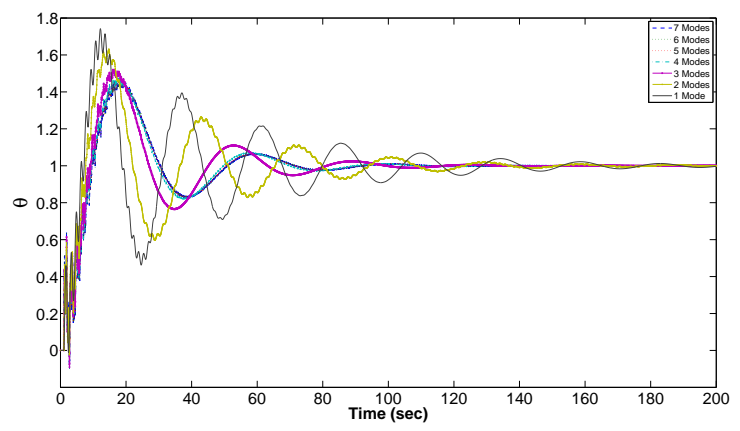

Fig. 6. Position response of the robotic arm system corresponding to a unit step change in reference position. Here, different numbers of modes are used in the plant model.

Also, $J^{T} \bar{G}(0) J=-0.309908135<0$. Thus, the conditions of Corollary 1 are satisfied.

To verify the performance of the closed loop system, we simulate the response of this system corresponding to a step change in the reference position of the robotic arm; see Fig. 5. This step response is shown in Fig. 6. Also, the corresponding response of the piezo sensor output $V_{s}$ is shown in Fig. 7. Here, the step responses were calculated using finite dimensional plant models defined in (47) for different numbers of modes, $\mathrm{n}=2,3 \ldots 7$.

To this end, we have used the proposed controller which is designed for the finite dimensional model with $n=1$ when applied to the plant with finite dimensional model where $\mathrm{n}=2,3 \ldots 7$ in order to check the performance and robustness of the proposed controller. In fact, the performance of the closed loop system is found to improve by increasing the number of modes; see Fig 6 and Fig 7.

Note that the controller parameters in (53) were chosen by process of trial and error to obtain good closed loop performance the case of the nominal plant model, $n=1$. An alternative approach, which would be useful in the case of a more complicated SNI controller structure, would be to use an optimization procedure to obtain the controller parameters; e.g., see [61].

\section{CONClusion}

In this paper, new stability results for the positive-feedback interconnection of negative imaginary systems have been derived. A new NI definition is presented, which allows for

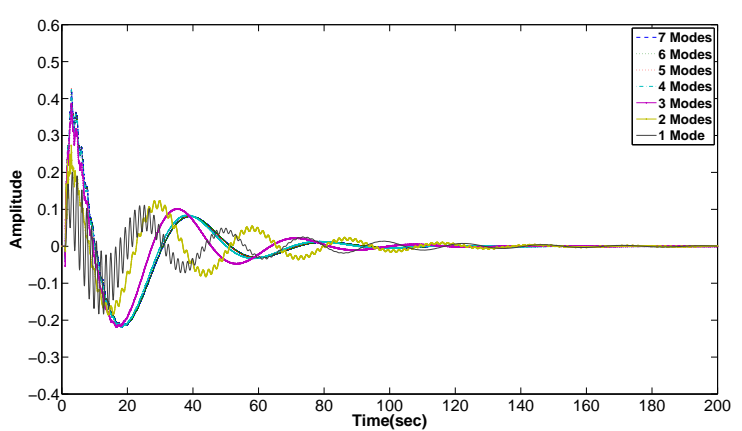

Fig. 7. Piezoelectric response sensor output $V_{s}$ response corresponding to a unit step change in reference position. Here, different numbers of modes are used in the plant model.

systems having free body dynamics to be considered as NI systems. This work can be used in controller design to allow for a broader class of NI systems than considered in previous work. The application of the main results in this paper has been illustrated via a case study involving the control of a flexible robotic arm.

\section{REFERENCES}

[1] D. G. Wilson, R. D. Robinett, G. G. Parker, and G. P. Starr, "Augmented sliding mode control for flexible link manipulators," Journal of Intelligent and Robotic Systems, vol. 34, no. 4, pp. 415-430, 2002.

[2] M. Harigae, I. Yamaguchi, T. Kasai, H. Igawa, and T. Suzuki, "Control of large space structures using GPS modal parameter identification and attitude and deformation estimation," Electronics and Communications in Japan, vol. 86, no. 4, pp. 63-71, 2003.

[3] B. Bhikkaji and S. Moheimani, "Fast scanning using piezoelectric tube nanopositioners: A negative imaginary approach," in Proc. IEEE/ASME Int. Conf. Advanced Intelligent Mechatronics AIM, Singapore, July 2009, pp. $274-279$.

[4] I. A. Mahmood, S. O. R. Moheimani, and B. Bhikkaji, "A new scanning method for fast atomic force microscopy," IEEE Transactions on Nanotechnology, vol. 10, no. 2, pp. 203-216, 2011.

[5] S. Salapaka, A. Sebastian, J. P. Cleveland, and M. V. Salapaka, "High bandwidth nano-positioner: A robust control approach," Review of Scientific Instruments, vol. 73, no. 9, pp. 3232-3241, 2002.

[6] J. R. van Hulzen, G. Schitter, P. M. J. Van den Hof, and J. van Eijk, "Modal actuation for high bandwidth nano-positioning," in Proc. American Control Conference, Baltimore, Maryland, USA, July 2010, pp. 6525-6530.

[7] S. Devasia, E. Eleftheriou, and S. O. R. Moheimani, "A survey of control issues in nanopositioning," IEEE Transactions on Control Systems Technology, vol. 15, no. 5, pp. 802-823, 2007.

[8] I. M. Diaz, E. Pereira, and P. Reynolds, "Integral resonant control scheme for cancelling human-induced vibrations in light-weight pedestrian structures," Structural Control and Health Monitoring, vol. 19, no. 1, pp. 55-69, 2012.

[9] W. Ray, "Some recent applications of distributed parameter systems theory a survey," Automatica, vol. 14, no. 3, pp. $281-287$, 1978.

[10] R. Curtain and K. Morris, "Transfer functions of distributed parameter systems: A tutorial," Automatica, vol. 45, no. 5, pp. 1101 - 1116, 2009.

[11] M. Demetriou and R. Smith, Research Directions in Distributed Parameter Systems, ser. Frontiers in applied mathematics. Society for Industrial and Applied Mathematics (SIAM, 3600 Market Street, Floor 6, Philadelphia, PA 19104), 2003.

[12] A. Preumont, Vibration Control of Active Structures: An Introduction, N. Jacobs, Ed. Springer, 2011.

[13] J. L. Fanson and T. K. Caughley, "Positive position feedback control for large space structures," AIAA Journal, vol. 28, no. 4, pp. 717-724, Apr. 1990.

[14] I. R. Petersen and A. Lanzon, "Feedback control of negative imaginary systems," IEEE Control System Magazine, vol. 30, no. 5, pp. 54-72, 2010. 
[15] M. J. Balas, "Direct velocity feedback control of large space structures1," AIAA Journal of Guidance and Control, vol. 2, no. 3, pp. 252-253, MayJune 1979.

[16] A. Lanzon and I. R. Petersen, "Stability robustness of a feedback interconnection of systems with negative imaginary frequency response," IEEE Transactions on Automatic Control, vol. 53, no. 4, pp. 1042-1046, 2008.

[17] B. D. O. Anderson and S. Vongpanitlerd, Network Analysis and Synthesis: A Modern Systems Approach. Englewood Cliffs, N.J., USA: Prentice-Hall, 1973.

[18] B. Brogliato, R. Lozano, B. Maschke, and O. Egeland, Dissipative Systems Analysis and Control, 2nd ed., ser. Communications and Control Engineering. London, UK: Springer, 2007.

[19] J. Dong, S. M. Salapaka, and P. M. Ferreira, "Robust MIMO control of a parallel kinematics nano-positioner for high resolution high bandwidth tracking and repetitive tasks," in Proc. 46th IEEE Conf. Decision and Control, New Orleans, LA, Dec 2007, pp. 4495-4500.

[20] A. Sebastian and S. M. Salapaka, "Design methodologies for robust nano-positioning," IEEE Transactions on Control Systems Technology, vol. 13, no. 6, pp. 868-876, 2005.

[21] Y. Michellod, P. Mullhaupt, and D. Gillet, "Strategy for the control of a dual-stage nano-positioning system with a single metrology," in Proc. IEEE Conf. Robotics, Automation and Mechatronics, Bangkok, June 2006, pp. 1-8.

[22] R. K. Messenger, Q. T. Aten, T. W. McLain, and L. L. Howell, "Piezoresistive feedback control of a MEMS thermal actuator," Journal of Microelectromechanical Systems, vol. 18, no. 6, pp. 1267-1278, 2009.

[23] K. El Rifai, O. El Rifai, and K. Youcef-Toumi, "On dual actuation in atomic force microscopes," in Proc. American Control Conference, vol. 4, 2004, pp. 3128-3133.

[24] Y. K. Yong, B. Ahmed, and S. O. R. Moheimani, "Atomic force microscopy with a 12-electrode piezoelectric tube scanner," Review of Scientific Instruments, vol. 81, no. 3, p. 033701, March 2010.

[25] C. Cai and G. Hagen, "Stability analysis for a string of coupled stable subsystems with negative imaginary frequency response," IEEE Transactions on Automatic Control, vol. 55, no. 8, pp. 1958-1963, Aug. 2010.

[26] C. J. Goh and T. K. Caughley, "On the stability problem caused by finite actuator dynamics in the collocated control of large space structures," International Journal of Control, vol. 41, no. 3, pp. 787-802, Mar. 1995.

[27] E. Pereira, S. S. Aphale, V. Feliu, and S. O. R. Moheimani, "Integral resonant control for vibration damping and precise tip-positioning of a single-link flexible manipulator," IEEE/ASME Transactions on Mechatronics, vol. 16, no. 2, pp. 232-240, 2011.

[28] D. Halim and S. O. R. Moheimani, "Spatial resonant control of flexible structures-application to a piezoelectric laminate beam," IEEE Transactions on Control Systems Technology, vol. 9, no. 1, pp. 37-53, 2001.

[29] I. A. Mahmood, S. O. R. Moheimani, and B. Bhikkaji, "Precise tip positioning of a flexible manipulator using resonant control," IEEE /ASME Transactions on Mechatronics, vol. 13, no. 2, pp. 180-186, 2008.

[30] B. Ahmed and H. Pota, "Dynamic compensation for control of a rotary wing UAV using positive position feedback," Journal of Intelligent and Robotic Systems, vol. 61, no. 1-4, pp. 43-56, 2011.

[31] B. Bhikkaji, S. O. R. Moheimani, and I. R. Petersen, "A negative imaginary approach to modeling and control of a collocated structure," IEEE/ASME Transactions on Mechatronics, vol. 17, no. 4, pp. 717-727, 2012.

[32] A. van der Schaft, "Positive feedback interconnection of Hamiltonian systems," in Proceedings of the 50th IEEE Conference on Decision and Control and European Control Conference (CDC-ECC), Orlando, FL, USA, Dec 2011

[33] M. R. Opmeer, "Infinite-dimensional negative imaginary systems," IEEE Transactions on Automatic Control, vol. 56, no. 12, pp. 2973-2976, 2011.

[34] J. Xiong, I. R. Petersen, and A. Lanzon, "A negative imaginary lemma and the stability of interconnections of linear negative imaginary systems," IEEE Transactions on Automatic Control, vol. 55, no. 10, pp. 2342-2347, 2010.

[35] Z. Song, A. Lanzon, S. Patra, and I. Petersen, "Towards controller synthesis for systems with negative imaginary frequency response," IEEE Transactions on Automatic Control, vol. 55, no. 6, pp. 1506-1511, June 2010.

[36] Z. Song, A. Lanzon, S. Patra, and I. R. Petersen, "A negative-imaginary lemma without minimality assumptions and robust state-feedback synthesis for uncertain negative-imaginary systems," Systems \& Control Letters, vol. 61, no. 12, pp. 1269 - 1276, 2012. [Online]. Available: http://www.sciencedirect.com/science/article/pii/S0167691112001557
[37] S. Engelken, S. Patra, A. Lanzon, and I. R. Petersen, "Stability analysis of negative imaginary systems with real parametric uncertainty - the SISO case," IET Control Theory and Applications, vol. 4, no. 11, pp. $2631-2638,2010$.

[38] S. Patra and A. Lanzon, "Stability analysis of interconnected systems with mixed negative-imaginary and small-gain properties," IEEE Transactions on Automatic Control, vol. 56, no. 6, pp. 1395 - 1400, 2011.

[39] J. Xiong, I. R. Petersen, and A. Lanzon, "Finite frequency negative imaginary systems,," IEEE Transactions on Automatic Control, vol. 57, pp. 2917-2922, 2012.

[40] S. Rao, "An algebraic approach to the realization of lossless negative imaginary behaviors," SIAM Journal on Control and Optimization, vol. 50, no. 3, pp. 1700-1720, 2012.

[41] P. Benner and M. Voigt, "Spectral characterization and enforcement of negative imaginariness for descriptor systems," April 2012, not. [Online]. Available: http://www.mpi-magdeburg.mpg.de/preprints/

[42] D. Angeli, "Systems with counterclockwise input-output dynamics," IEEE Transactions on Automatic Control, vol. 51, no. 7, pp. 1130-1143, 2006.

[43] — "Multistability in systems with counter-clockwise input output dynamics," IEEE Transactions on Automatic Control, vol. 52, no. 4, pp. $596-609,2007$.

[44] A. K. Padthe, J. H. Oh, and D. S. Bernstein, "Counterclockwise dynamics of a rate-independent semilinear Duhem model," in Proceedings of the 44th IEEE Conference on Decision and Control, Seville, Spain, December 2005, pp. 8000-8005.

[45] P. Hughes, Spacecraft Attitude Dynamics. New York: Wiley, 1986.

[46] T. Gustafsson, "On the design and implementation of a rotary crane controller," European Journal of Control, vol. 2, no. 2, pp. 166-175, 1996.

[47] S. B. Choi, S. S. Cho, H. C. Shin, and H. K. Kim, "Quantitative feedback theory control of a single-link flexible manipulator featuring piezoelectric actuator and sensor," Smart Materials and Structures, vol. 8, no. 3, pp. 338-349, 1999.

[48] Y. Li, R. Horowitz, and R. Evans, "Vibration control of a PZT actuated suspension dual-stage servo system using a PZT sensor," IEEE Transactions on Magnetics, vol. 39, no. 2, pp. 932 - 937, 2003.

[49] S.-M. Suh, C. C. Chung, and S.-H. Lee, "Discrete-time LQG/LTR dualstage controller design in magnetic disk drives," IEEE Transactions on Magnetics, vol. 37, no. 4, pp. 1891 - 1895, 2001.

[50] T. B. Goh, Z. Li, B. M. Chen, T. H. Lee, and T. Huang, "Design and implementation of a hard disk drive servo system using robust and perfect tracking approach," IEEE Transactions on Control Systems Technology, vol. 9, no. 2, pp. 221-233, 2001.

[51] S. Devasia, "Time-optimal control with pre/post actuation for dual-stage systems," IEEE Transactions on Control Systems Technology, vol. 20, no. 2, pp. 323-334, 2012.

[52] C. La-orpacharapan and L. Y. Pao, "Shaped time-optimal feedback control for disk-drive systems with back-electromotive force," IEEE Transactions on Magnetics, vol. 40, no. 1, pp. 85-96, 2004.

[53] M. A. Mabrok, A. G. Kallapur, I. R. Petersen, and A. Lanzon, "A stability result on the feedback interconnection of negative imaginary systems with poles at the origins," in Proceedings of the 2012 Australian Control Conference, Sydney, Australia, 2012.

[54] _ _A new stability result for the feedback interconnection of negative imaginary systems with a pole at the origin." in Proceedings of the Conference Decision Control-European Control Conference, Orlando, Florida, USA. IEEE, 2011, pp. 3753-3757.

[55] K. Zhou, J. C. Doyle, and K. Glover, Robust and Optimal Control. Upper Saddle River, NJ: Prentice-Hall, Inc., 1996.

[56] M. Koeber, U. Schfer, and U. Karlsruhe, "The unique square root of a positive semidefinite matrix," International Journal of Mathematical Education in Science and Technology, vol. 37, no. 8, pp. 990-992, 2006

[57] H. R. Pota and T. E. Alberts, "Multivariable transfer functions for a slewing piezoelectric laminate beam," Journal of Dynamic Systems, Measurements and Control, vol. 117, no. 2, pp. 352 - 359, 1995.

[58] T. Alberts, T. DuBois, and H. Pota, "Experimental verification of transfer functions for a slewing piezoelectric laminate beam," Control Engineering Practice, vol. 3, no. 2, pp. 163 - 170, 1995.

[59] E. Schmitz, "Experiments on the End-Point Position Control of a Very Flexible One-Link Manipulator," PhD, Stanford University, Stanford, CA 94305, June 1985, also published as SUDAAR 548.

[60] L. Meirovitch, Elements of Vibrational Analysis. USA: McGraw-Hill, 1975.

[61] S. O. R. Moheimani, B. J. G. Vautier, and B. Bhikkaji, "Experimental implementation of extended multivariable ppf control on an active 
structure," IEEE Transactions on Control Systems Technology, vol. 14, no. 3, pp. 443-455, 2006.

[62] B. Chen, $H_{\infty}$ Control and Its Applications, ser. Lecture Notes in Control and Information Sciences. Springer, New York, London, 1998

\section{APPENDIX A}

In this appendix, we present state space results (some of which are of independent interest) using a particular state space representation of the plant transfer function matrix $G(s)$. The first stability result is Theorem 5 . We will subsequently use Theorem 5 to prove Theorem 1 . We also present a number of corollaries which will be used to prove the remaining results of the paper.

We first consider an NI square transfer function matrix $G(s)$ with a minimal state space realization of the form

$$
\begin{aligned}
& \dot{x}(t)=A x(t)+B u(t), \\
& y(t)=C x(t),
\end{aligned}
$$

where, $A \in \mathbb{R}^{n \times n}, B \in \mathbb{R}^{n \times m}, C \in \mathbb{R}^{m \times n}$, and

$$
A=\left[\begin{array}{ccc}
A_{1} & 0 & 0 \\
0 & A_{2} & 0 \\
0 & 0 & A_{3}
\end{array}\right] ; B=\left[\begin{array}{l}
B_{1} \\
B_{2} \\
B_{3}
\end{array}\right] ; C=\left[\begin{array}{lll}
C_{1} & C_{2} & C_{3}
\end{array}\right],
$$

$A_{1} \in \mathbb{R}^{n_{1} \times n_{1}}, A_{2} \in \mathbb{R}^{n_{2} \times n_{2}}, A_{3} \in \mathbb{R}^{2 k \times 2 k}, B_{1} \in$ $\mathbb{R}^{n_{1} \times m}, B_{2} \in \mathbb{R}^{n_{2} \times m}, B_{3}=\left[\begin{array}{c}B_{3 a} \\ B_{3 b}\end{array}\right], B_{3 a} \in$ $\mathbb{R}^{k \times m}, B_{3 b} \in \mathbb{R}^{k \times m}, C_{1} \in \mathbb{R}^{m \times n_{1}}, C_{2} \in \mathbb{R}^{m \times n_{2}}, C_{3}=$ $\left[\begin{array}{ll}C_{3 a} & C_{3 b}\end{array}\right], C_{3 a} \in \mathbb{R}^{m \times k}, C_{3 b} \in \mathbb{R}^{m \times k}, A_{1}$ is nonsingular, $A_{2}=0$, and

$$
A_{3}=\left[\begin{array}{cc}
0 & I_{k \times k} \\
0 & 0
\end{array}\right] \text {. }
$$

We also consider an SNI transfer function matrix $\bar{G}(s)$ with a minimal state space realization

$$
\begin{aligned}
& \dot{x}(t)=\bar{A} x(t)+\bar{B} u(t), \\
& y(t)=\bar{C} x(t)+\bar{D} u(t),
\end{aligned}
$$

where $\bar{A} \in \mathbb{R}^{\bar{n} \times \bar{n}}, \bar{B} \in \mathbb{R}^{\bar{n} \times m}, \bar{C} \in \mathbb{R}^{m \times \bar{n}}$, and $\bar{D} \in \mathbb{R}^{m \times m}$.

Remark 2: We allow any of the matrices in these models to have zero dimensions. In sequel, any matrix with zero dimension is regarded as being of full rank.

The corresponding transfer function matrix for the state space realization (54)-(55) is given as follows:

$$
\begin{aligned}
G(s)= & C_{1}\left(s I-A_{1}\right)^{-1} B_{1}+C_{2}\left(s I-A_{2}\right)^{-1} B_{2} \\
& +C_{3}\left(s I-A_{3}\right)^{-1} B_{3} \\
= & C_{1}\left(s I-A_{1}\right)^{-1} B_{1}+\frac{C_{2} B_{2}+C_{3} B_{3}}{s}+\frac{C_{3 a} B_{3 b}}{s^{2}} .
\end{aligned}
$$

The following theorem provides a necessary and sufficient condition for the stability of the positive-feedback interconnection between the NI transfer function matrix $G(s)$, with state space realization (54)-(55), and the SNI transfer function matrix $\bar{G}(s)$, with state space realization (57). In order to present this theorem, we define the following matrix

$$
N=\mathcal{P}\left(\bar{G}(0),\left[\begin{array}{ll}
C_{2} & C_{3 a}
\end{array}\right]\right) .
$$

Also, the matrix

$$
\Xi=\left[\begin{array}{c}
C_{2}^{T} \\
C_{3 a}^{T}
\end{array}\right] \bar{G}(0)\left[\begin{array}{ll}
C_{2} & C_{3 a}
\end{array}\right]
$$

is assumed to be non-singular. In addition, we will use the following condition in the theorem which follows:

$$
\left[\begin{array}{c}
C_{2}^{T} \\
C_{3 a}^{T}
\end{array}\right] \bar{G}(0)\left[\begin{array}{ll}
C_{2} & C_{3 a}
\end{array}\right]<0
$$

Also, for the case in which $N$ is positive semidefinite, we will use the condition

$$
I+N^{\frac{1}{2}} C_{1} A_{1}^{-1} B_{1} N^{\frac{1}{2}}-N^{\frac{1}{2}} C_{3 b} P_{2}^{-1} C_{3 b}^{T} N^{\frac{1}{2}}>0,
$$

where $P_{2}=C_{3 a}^{T} B_{3 b}^{T}\left(B_{3 b} B_{3 b}^{T}\right)^{-1}$, which will be shown to be symmetric and positive definite in Lemma 3. Moreover, for the case in which $N$ is negative semidefinite, we will use the condition

$$
\operatorname{det}\left(I-\tilde{N} C_{1} A_{1}^{-1} B_{1} \tilde{N}+\tilde{N} C_{3 b} P_{2}^{-1} C_{3 b}^{T} \tilde{N}\right) \neq 0,
$$

where $\tilde{N}=(-N)^{\frac{1}{2}}$.

Theorem 5: Suppose that $k \neq 0$ and the matrix $\Xi$ in (60) is non-singular. Also, suppose that the transfer function matrix $G(s)$, with the minimal state space realization (54), is NI and the transfer function matrix $\bar{G}(s)$, with the minimal state space realization (57), is SNI. If $N$ is positive semidefinite, then the closed-loop positive-feedback interconnection between $G(s)$ and $\bar{G}(s)$ is internally stable if and only if conditions (61) and (62) are satisfied. Also, if $N$ is negative semidefinite, then the closed-loop positive-feedback interconnection between $G(s)$ and $\bar{G}(s)$ is internally stable if and only if conditions (61) and (63) are satisfied.

The proof of this theorem is given at the end of this appendix.

Corollary 3: Suppose that the matrix $\Xi$ in (60) is nonsingular and the matrix $N$ in (59) satisfies $N\left[\begin{array}{ll}C_{1} & C_{3 b}\end{array}\right]=0$. Also suppose that the transfer function matrix $G(s)$, with the minimal state space realization (54), is NI and the transfer function matrix $\bar{G}(s)$, with the minimal state space realization (57), is SNI. Then the closed-loop positive-feedback interconnection between $G(s)$ and $\bar{G}(s)$ is internally stable if and only if condition (61) is satisfied.

The proof of this corollary is given at the end of this appendix.

The following corollary considers the case when $n_{2}=0$ and $k \neq 0$; i.e., the matrix $A$ in (55) has the block diagonal form $A=\left[\begin{array}{cc}A_{1} & 0 \\ 0 & A_{3}\end{array}\right]$. In the case when $n_{2}=0$, the matrix $N$ in (59) will be given by

$$
N=\mathcal{P}\left(\bar{G}(0), C_{3 a}\right),
$$

where we assume that $C_{3 a}^{T} \bar{G}(0) C_{3 a}$ is non-singular.

We will use the following conditions in the next corollary which correspond to conditions (61)-(63) in Theorem 5. The first condition to be considered is

$$
C_{3 a}^{T} \bar{G}(0) C_{3 a}<0 .
$$


Also, for the case in which $N$ is positive semidefinite, we will use the condition

$$
I+N^{\frac{1}{2}} C_{1} A_{1}^{-1} B_{1} N^{\frac{1}{2}}-N^{\frac{1}{2}} C_{3 b} P_{2}^{-1} C_{3 b}^{T} N^{\frac{1}{2}}>0,
$$

where $P_{2}=C_{3 a}^{T} B_{3 b}^{T}\left(B_{3 b} B_{3 b}^{T}\right)^{-1}$. Moreover, for the case in which $N$ is negative semidefinite, we will use the condition

$$
\operatorname{det}\left(I-\tilde{N} C_{1} A_{1}^{-1} B_{1} \tilde{N}+\tilde{N} C_{3 b} P_{2}^{-1} C_{3 b}^{T} \tilde{N}\right) \neq 0,
$$

where $\tilde{N}=(-N)^{\frac{1}{2}}$.

Corollary 4: Suppose that the matrix $C_{3 a}^{T} \bar{G}(0) C_{3 a}$ is nonsingular, $k \neq 0$, and $n_{2}=0$. Also, suppose that the transfer function matrix $G(s)$, with the minimal state space realization (54) is NI and the transfer function matrix $\bar{G}(s)$, with the minimal state space realization in (57), is SNI. If $N$ in (64) is positive semidefinite, then the closed-loop positive-feedback interconnection between $G(s)$ and $\bar{G}(s)$ is internally stable if and only if conditions (65) and (66) are satisfied. Also, if $N$ in (64) is negative semidefinite, then the closed-loop positivefeedback interconnection between $G(s)$ and $\bar{G}(s)$ is internally stable if and only if conditions (65) and (67) are satisfied.

The proof of this corollary is given at the end of this appendix.

The next corollary considers the case when $n \neq 0$ and $k=$ 0 ; i.e., the $A$ matrix in the minimal state realization of $G(s)$ (54)-(55) has the block diagonal form $A=\left[\begin{array}{cc}A_{1} & 0 \\ 0 & A_{2}\end{array}\right]$. In this case, when $n \neq 0$ and $k=0$, the matrix $N$ in (59) will be given by

$$
N=\mathcal{P}\left(\bar{G}(0), C_{2}\right)
$$

where the matrix $C_{2}^{T} \bar{G}(0) C_{2}$ is assumed to be non-singular.

We will use the following conditions in the next corollary which corresponds to conditions (61)-(63) in Theorem 5. The first condition to be considered is

$$
C_{2}^{T} \bar{G}(0) C_{2}<0 .
$$

Also, for the case in which $N$ in (68) is positive semidefinite, we will use the condition

$$
I+N^{\frac{1}{2}} C_{1} A_{1}^{-1} B_{1} N^{\frac{1}{2}}>0 .
$$

Moreover, for the case in which $N$ in (68) is negative semidefinite, we will use the condition

$$
\operatorname{det}\left(I-\tilde{N} C_{1} A_{1}^{-1} B_{1} \tilde{N}\right) \neq 0,
$$

where $\tilde{N}=(-N)^{\frac{1}{2}}$.

Corollary 5: Suppose that $C_{2}^{T} \bar{G}(0) C_{2}$ is non-singular, $n_{2} \neq 0$, and $k=0$. Also, suppose that the transfer function matrix $G(s)$, with the minimal state space realization (54) is $\mathrm{NI}$ and the transfer function matrix $\bar{G}(s)$, with the minimal state space realization in (57), is SNI. If $N$ in (68) is positive semidefinite, then the closed-loop positive-feedback interconnection between $G(s)$ and $\bar{G}(s)$ is internally stable if and only if conditions (69) and (70) are satisfied. Also, if $N$ in (68) is negative semidefinite, then the closed-loop positivefeedback interconnection between $G(s)$ and $\bar{G}(s)$ is internally stable if and only if conditions (69) and (71) are satisfied.

The proof of this corollary is given at the end of this appendix.

In order to prove Theorem 5 and Corollaries 3-5, we will use the following lemmas. First, Lemma 1 gives expressions for the quantities $G_{0}, G_{1}$ and $G_{2}$ in (5) in terms of the state space realization (54)-(55).

Lemma 1: Suppose that $G(s)$ has a minimal state space realization (54)-(55). Then the quantities $G_{0}, G_{1}$ and $G_{2}$ defined in (5) are given as follows:

$$
\begin{aligned}
& G_{2}=C_{3 a} B_{3 b}, \\
& G_{1}=C_{2} B_{2}+C_{3} B_{3}, \\
& G_{0}=-C_{1} A_{1}^{-1} B_{1} .
\end{aligned}
$$

Proof: This lemma follows immediately from (58).

Now, Lemmas 2, 3, 4 give some useful properties of the minimal state space realization (54)-(55).

Lemma 2: Suppose that the transfer function matrix $G(s)$ has a minimal state space realization (54)-(55). Then, the matrix $\left[\begin{array}{ll}C_{2} & C_{3 a}\end{array}\right]$ is of full column rank, and the matrix $\left[\begin{array}{c}B_{2} \\ B_{3 b}\end{array}\right]$ is of full row rank. Also $m \geq k+n_{2}$ and the subsystem with realization $\left[\begin{array}{c|c}A_{1} & B_{1} \\ \hline C_{1} & 0\end{array}\right]$ is minimal.

Proof: Since the state space realization $\left[\begin{array}{c|c}A & B \\ \hline C & 0\end{array}\right]$ is minimal, the pair $(A, C)$ is observable and the pair $(A, B)$ is controllable. Also, the corresponding observability matrix is given by

$$
O(A, C)=\left[\begin{array}{c}
C \\
C A \\
C A^{2} \\
\vdots \\
C A^{n-1}
\end{array}\right]=\left[\begin{array}{cccc}
C_{1} & C_{2} & C_{3 a} & C_{3 b} \\
C_{1} A_{1} & 0 & 0 & C_{3 a} \\
C_{1} A_{1}^{2} & 0 & 0 & 0 \\
\vdots & \vdots & \vdots & \vdots \\
C_{1} A_{1}^{n-1} & 0 & 0 & 0
\end{array}\right] .
$$

Since the pair $(A, C)$ is observable, it follows that the observability matrix $O(A, C)$ is of full rank. This implies that the pair $\left(A_{1}, C_{1}\right)$ is observable. Also, since the observability matrix $O(A, C)$ is of full rank, it follows that $C_{2}, C_{3 a}$ and $\left[\begin{array}{ll}C_{2} & C_{3 a}\end{array}\right]$ are of full rank. Furthermore, it follows that $m \geq k+n_{2}$. Similarly, since the pair $(A, B)$ is controllable, it follows that the corresponding controllability matrix

$$
\begin{aligned}
\mathcal{C}(A, B)= & {\left[\begin{array}{lllll}
B & A B & A^{2} B & \cdots & A^{n-1} B
\end{array}\right] } \\
= & {\left[\begin{array}{ccccc}
B_{1} & A_{1} B_{1} & A_{1}^{2} B_{1} & \cdots & A_{1}^{n-1} B_{1} \\
B_{2} & 0 & 0 & \cdots & 0 \\
B_{3 a} & B_{3 b} & 0 & \cdots & 0 \\
B_{3 b} & 0 & 0 & \cdots & 0
\end{array}\right], }
\end{aligned}
$$

is of full rank. Hence, the pair $\left(A_{1}, B_{1}\right)$ is controllable and the matrices $B_{2}, B_{3 b}$ and $\left[\begin{array}{c}B_{2} \\ B_{3 b}\end{array}\right]$ are of full rank. Also, since the pair $\left(A_{1}, C_{1}\right)$ is observable and the pair $\left(A_{1}, B_{1}\right)$ is controllable, it follows that $\left[\begin{array}{c|c}A_{1} & B_{1} \\ \hline C_{1} & 0\end{array}\right]$ is a minimal realization. 
Lemma 3: Suppose that the transfer function matrix $G(s)$, with the minimal state space realization (54)-(55), is NI. Then, there exist symmetric matrices $P_{1}>0, P_{2}>0$, and matrices $L_{1}, W$ such that

$$
\begin{aligned}
& P_{1} A_{1}+A_{1}^{T} P_{1}=-L_{1}^{T} L_{1}, \\
& P_{1} B_{1}-A_{1}^{T} C_{1}^{T}=-L_{1}^{T} W \\
& P_{2} B_{3 b}=C_{3 a}^{T}, \\
& C_{1} B_{1}+B_{1}^{T} C_{1}^{T}+C_{2} B_{2}+B_{2}^{T} C_{2}^{T}+C_{3} B_{3}+B_{3}^{T} C_{3}^{T} \\
& =W^{T} W .
\end{aligned}
$$

Furthermore,

$$
P_{2}=C_{3 a}^{T} B_{3 b}^{T}\left(B_{3 b} B_{3 b}^{T}\right)^{-1}
$$

and

$$
\begin{aligned}
G_{1}+G_{1}^{T} & =C_{2} B_{2}+B_{2}^{T} C_{2}^{T}+C_{3} B_{3}+B_{3}^{T} C_{3}^{T} \\
& =\left(W^{T}+C_{1} P_{1}^{-1} L_{1}^{T}\right)\left(W+L_{1} P_{1}^{-1} C_{1}^{T}\right) \geq 0
\end{aligned}
$$

Proof: Consider the transfer function matrix $G(s)$ with the minimal state space realization (54)-(55). Also, define the transfer function matrix $R(s)=s G(s)$. Using (58), it follows that

$$
\begin{aligned}
R(s)= & s C_{1}\left(s I-A_{1}\right)^{-1} B_{1}+\frac{C_{3 a} B_{3 b}}{s}+C_{2} B_{2}+C_{3} B_{3} \\
= & C_{1} A_{1}\left(s I-A_{1}\right)^{-1} B_{1}+C_{1} B_{1}+\frac{C_{3 a} B_{3 b}}{s} \\
& +C_{2} B_{2}+C_{3} B_{3} .
\end{aligned}
$$

This implies that $R(s)$ has a state space realization $\left[\begin{array}{l|l}A_{r} & B_{r} \\ \hline C_{r} & D_{r}\end{array}\right]$ where $A_{r}=\left[\begin{array}{cc}A_{1} & 0 \\ 0 & 0\end{array}\right], B_{r}=\left[\begin{array}{c}B_{1} \\ B_{3 b}\end{array}\right], C_{r}=$ $\left[\begin{array}{ll}C_{1} A_{1} & C_{3 a}\end{array}\right]$ and $D_{r}=C_{1} B_{1}+C_{2} B_{2}+C_{3} B_{3}$. Using the same argument as in the proof of Lemma 2, it follows that the rank of the matrix formed from the first and last columns in $O\left(A_{r}, C_{r}\right)$ is equal to the rank of the matrix formed from the first and third columns in (56), where, $A_{1}$ is invertible. This implies that the matrix $O\left(A_{r}, C_{r}\right)$ is of full rank; i.e., the pair $\left(A_{r}, C_{r}\right)$ is observable. Similarly, the pair $\left(A_{r}, B_{r}\right)$ is controllable. This implies that the state space realization $\left[\begin{array}{l|l}A_{r} & B_{r} \\ \hline C_{r} & D_{r}\end{array}\right]$ is minimal.

We now show that $R(s)$ is positive real; e.g., see page 47 in [18] for a definition of positive real transfer function matrices. Since $G(s)$ is NI, it follows that $j\left(G(j \omega)-G(j \omega)^{*}\right) \geq 0$, for all $\omega>0$ such that $j \omega$ is not a pole of $G(s)$. Then given any such $\omega>0, R(j \omega)+R(j \omega)^{*}=j \omega\left(G(j \omega)-G(j \omega)^{*}\right) \geq$ 0 , and $\overline{\left(R(j \omega)+R(j \omega)^{*}\right)} \geq 0$. This implies that $R(-j \omega)+$ $R(-j \omega)^{*} \geq 0$ for all $\omega>0$, since $\overline{R(j \omega)}=R(-j \omega)$. Hence, $R(j \omega)+R(j \omega)^{*} \geq 0$ for all $\omega<0$ such that $j \omega$ is not a pole of $G(s)$. Therefore , $R(j \omega)+R(j \omega)^{*} \geq 0$ for all $\omega \in(-\infty, \infty)$ such that $j \omega$ is not a pole of $G(s)$.

Now, consider the case where $j \omega_{0}$ is a pole of $G(s)$ and $\omega_{0}=0$. In the case where $C_{3 a} B_{3 b}=0$, the transfer function matrix $R(s)=C_{1} A_{1}\left(s I-A_{1}\right)^{-1} B_{1}+C_{1} B_{1}+C_{2} B_{2}+C_{3} B_{3}$ will have no pole at the origin. This implies that $R(0)$ is finite.
Since $R(j \omega)+R(j \omega)^{*} \geq 0$ for all $\omega>0$ such that $j \omega$ is not a pole of $G(s)$ and $R(j \omega)$ is continuous at $\omega=0$, this implies that $R(0)+R(0)^{*} \geq 0$. In the case where $C_{3 a} B_{3 b} \neq 0$, the transfer function matrix $R(s)$ is as given in (81). Since $G(s)$ is NI, then $\lim _{s \rightarrow 0} s^{2} G(s) \geq 0$ which implies that $\lim _{s \rightarrow 0} s R(s) \geq 0$.

If $j \omega_{0}$ is a pole of $G(s)$ and $\omega_{0}>0$, then $G(s)$ can be factored as $\frac{1}{s^{2}+\omega_{0}^{2}} F(s)$, which according to the definition for NI systems implies that the residue matrix $K_{0}=\frac{1}{2 \omega_{0}} F\left(j \omega_{0}\right)$ is positive semidefinite Hermitian. Hence, $F\left(j \omega_{0}\right)=F\left(j \omega_{0}\right)^{*} \geq$ 0 . Now, the residue matrix of $R(s)$ at $j \omega_{0}$ with $\omega_{0}>0$ is given by,

$$
\begin{aligned}
\lim _{s \longrightarrow j \omega_{0}}\left(s-j \omega_{0}\right) R(s) & =\lim _{s \longrightarrow j \omega_{0}}\left(s-j \omega_{0}\right) s G(s), \\
& =\lim _{s \longrightarrow j \omega_{0}}\left(s-j \omega_{0}\right) s \frac{1}{s^{2}+\omega_{0}^{2}} F(s), \\
& =\frac{1}{2} F\left(j \omega_{0}\right)
\end{aligned}
$$

which is positive semidefinite Hermitian. Hence, we can conclude that $R(s)$ is positive real; see page 47 in [18]. Using the KYP lemma (e.g., see Lemma 3.1 in [18]), it now follows that there exist matrices $P_{r}>0, L$ and $W$ such that

$$
\begin{aligned}
P_{r} A_{r}+A_{r}^{T} P_{r} & =-L^{T} L, \\
P_{r} B_{r}-C_{r}^{T} & =-L^{T} W, \\
D_{r}+D_{r}^{T} & =W^{T} W .
\end{aligned}
$$

If we write $P_{r}=\left[\begin{array}{cc}P_{1} & P_{12} \\ P_{12}^{T} & P_{2}\end{array}\right]$ and $L=\left[\begin{array}{ll}L_{1} & L_{2}\end{array}\right]$, it follows from (82) that

$$
\begin{aligned}
& {\left[\begin{array}{cc}
P_{1} & P_{12} \\
P_{12}^{T} & P_{2}
\end{array}\right]\left[\begin{array}{cc}
A_{1} & 0 \\
0 & 0
\end{array}\right]+\left[\begin{array}{cc}
A_{1}^{T} & 0 \\
0 & 0
\end{array}\right]\left[\begin{array}{cc}
P_{1} & P_{12} \\
P_{12}^{T} & P_{2}
\end{array}\right] } \\
= & -\left[\begin{array}{c}
L_{1}^{T} \\
L_{2}^{T}
\end{array}\right]\left[\begin{array}{ll}
L_{1} & L_{2}
\end{array}\right], \\
\Leftrightarrow & {\left[\begin{array}{cc}
P_{1} A_{1}+A_{1}^{T} P_{1} & A_{1}^{T} P_{12} \\
P_{12}^{T} A_{1} & 0
\end{array}\right]=-\left[\begin{array}{ll}
L_{1}^{T} L_{1} & L_{1}^{T} L_{2} \\
L_{2}^{T} L_{1} & L_{2}^{T} L_{2}
\end{array}\right] . }
\end{aligned}
$$

Hence $L_{2}=0$ and since $A_{1}$ is a nonsingular matrix, it also follows that $P_{12}=0$. Also, (83) implies that (75) is satisfied. From (82), it follows that

$$
\left[\begin{array}{cc}
P_{1} & 0 \\
0 & P_{2}
\end{array}\right]\left[\begin{array}{c}
B_{1} \\
B_{3 b}
\end{array}\right]-\left[\begin{array}{c}
A_{1}^{T} C_{1}^{T} \\
C_{3 a}^{T}
\end{array}\right]=-\left[\begin{array}{c}
L_{1}^{T} \\
0
\end{array}\right] W,
$$

which implies (76) and (77). Lemma 2 implies that $B_{3 b}$ is of full rank and hence, (77) implies that (79) is also satisfied. From (82), it follows that (78) holds. Also, using (76), we can write $B_{1}$ as,

$$
B_{1}=P_{1}^{-1}\left(A_{1}^{T} C_{1}^{T}-L_{1}^{T} W\right) .
$$

Substituting this and (75) into (78), it follows that

$$
\begin{aligned}
& C_{2} B_{2}+B_{2}^{T} C_{2}^{T}+C_{3} B_{3}+B_{3}^{T} C_{3}^{T} \\
& =\left(W^{T}+C_{1} P_{1}^{-1} L_{1}^{T}\right)\left(W+L_{1} P_{1}^{-1} C_{1}^{T}\right) \geq 0 .
\end{aligned}
$$

Using (74) in Lemma 1, this implies (80). This completes the proof. 
Lemma 4: Suppose that the transfer function matrix $G(s)$ with the minimal state space realization (54)-(55) is NI. Then, there exists an invertible matrix $R_{d}$ such that $\left[\begin{array}{c}B_{2} \\ B_{3 b}\end{array}\right]=$ $R_{d}\left[\begin{array}{c}C_{2}^{T} \\ C_{3 a}^{T}\end{array}\right]$. Also, if $x \in \mathcal{N}\left(\left[\begin{array}{c}C_{2}^{T} \\ C_{3 a}^{T}\end{array}\right]\right)$, then $x \in \mathcal{N}\left(B_{3 a}+\right.$ $P_{2}^{-1} C_{3 b}^{T}$, where the matrix $P_{2}$ is defined as in Lemma 3 . Here, $\mathcal{N}(\cdot)$ denotes as the null space of a matrix.

Proof: Suppose that $x \in \mathcal{N}\left(\left[\begin{array}{c}C_{2}^{T} \\ C_{3 a}^{T}\end{array}\right]\right)$. It follows that $\left[\begin{array}{l}C_{2}^{T} \\ C_{3 a}^{T}\end{array}\right] x=0$. Hence using (77) in Lemma 3 , it follows that there exists a matrix $P_{2}>0$ such that $P_{2} B_{3 b} x=0$. Therefore, $x^{T} C_{2}=0, x^{T} C_{3 a}=0$ and $B_{3 b} x=0$. Hence, using (74) it follows that

$$
x^{T}\left(G_{1}+G_{1}^{T}\right) x=x^{T} G_{1} x+x^{T} G_{1}^{T} x .
$$

Using the fact that $x^{T} G_{1} x$ is a scalar, this implies

$$
\begin{aligned}
& x^{T}\left(G_{1}+G_{1}^{T}\right) x=2 x^{T} G_{1} x \\
\Rightarrow & 2 x^{T}\left(C_{2} B_{2}+C_{3 a} B_{3 a}+C_{3 b} B_{3 b}\right) x=0, \\
\Rightarrow & \left(G_{1}+G_{1}^{T}\right) x=0,
\end{aligned}
$$

since, $G_{1}+G_{1}^{T} \geq 0$ using (80) in Lemma 3. Hence,

$$
\begin{aligned}
& \left(C_{2} B_{2}+C_{3 a} B_{3 a}+B_{3 b}^{T} C_{3 b}^{T}\right) x=0, \\
\Rightarrow & \left(C_{2} B_{2}+C_{3 a} B_{3 a}+C_{3 a} P_{2}^{-1} C_{3 b}^{T}\right) x=0,
\end{aligned}
$$

using (77) in Lemma 3. Therefore,

$$
\begin{aligned}
& {\left[\begin{array}{ll}
C_{2} & C_{3 a}
\end{array}\right]\left[\begin{array}{c}
B_{2} \\
B_{3 a}+P_{2}^{-1} C_{3 b}^{T}
\end{array}\right] x=0, } \\
\Rightarrow & {\left[\begin{array}{c}
B_{2} \\
B_{3 a}+P_{2}^{-1} C_{3 b}^{T}
\end{array}\right] x=0, }
\end{aligned}
$$

since $\left[\begin{array}{ll}C_{2} & C_{3 a}\end{array}\right]$ is full rank using Lemma 2. Therefore,

$$
B_{2} x=0 \text { and }\left(B_{3 a}+P_{2}^{-1} C_{3 b}^{T}\right) x=0 .
$$

This implies that $x \in \mathcal{N}\left(B_{3 a}+P_{2}^{-1} C_{3 b}^{T}\right)$. Thus, we have established the second part of the lemma. Also since $B_{2} x=0$ and $B_{3 b} x=0$, it follows that $\left[\begin{array}{c}B_{2} \\ B_{3 b}\end{array}\right] x=0$. This implies that if $x \in \mathcal{N}\left(\left[\begin{array}{c}C_{2}^{T} \\ C_{3 a}^{T}\end{array}\right]\right)$, then $x \in \mathcal{N}\left(\left[\begin{array}{c}B_{2} \\ B_{3 b}\end{array}\right]\right)$.

Similarly, suppose that $x \in \mathcal{N}\left(\left[\begin{array}{c}B_{2} \\ B_{3 b}\end{array}\right]\right)$, and hence $\left[\begin{array}{c}B_{2} \\ B_{3 b}\end{array}\right] x=0$. Therefore, $B_{2} x=0, B_{3 b} x=0$ and $C_{3 a}^{T} x=0$. Hence using (73) in Lemma 1, it follows that

$$
\begin{aligned}
& x^{T}\left(G_{1}+G_{1}^{T}\right) x=2 x^{T} G_{1} x, \\
\Rightarrow & 2 x^{T}\left(C_{2} B_{2}+C_{3 a} B_{3 a}+C_{3 b} B_{3 b}\right) x=0, \\
\Rightarrow & x^{T}\left(G_{1}+G_{1}^{T}\right)=0,
\end{aligned}
$$

since, $G_{1}+G_{1}^{T} \geq 0$ using (80) in Lemma 3. Therefore,

$$
\begin{aligned}
& x^{T}\left(C_{2} B_{2}+C_{3 b} B_{3 b}+B_{3 a}^{T} C_{3 a}^{T}\right)=0, \\
\Rightarrow & x^{T}\left(C_{2} B_{2}+C_{3 b} B_{3 b}+B_{3 a}^{T} P_{2} B_{3 b}\right)=0,
\end{aligned}
$$

using (77) in Lemma 3. Therefore,

$$
\begin{aligned}
& x^{T}\left[\begin{array}{ll}
C_{2} & C_{3 b}+B_{3 a}^{T} P_{2}
\end{array}\right]\left[\begin{array}{c}
B_{2} \\
B_{3 b}
\end{array}\right]=0, \\
\Rightarrow & x^{T}\left[\begin{array}{ll}
C_{2} & C_{3 b}+B_{3 a}^{T} P_{2}
\end{array}\right]=0,
\end{aligned}
$$

since $\left[\begin{array}{c}B_{2} \\ B_{3 b}\end{array}\right]$ is full rank using Lemma 2. Therefore,

$$
\begin{aligned}
& x^{T} C_{2}=0, \\
\Rightarrow & x^{T}\left[\begin{array}{ll}
C_{2} & C_{3 a}
\end{array}\right]=0 .
\end{aligned}
$$

Therefore, $x \in \mathcal{N}\left(\left[\begin{array}{c}C_{2}^{T} \\ C_{3 a}^{T}\end{array}\right]\right)$. Hence,

$$
\mathcal{N}\left(\left[\begin{array}{c}
B_{2} \\
B_{3 b}
\end{array}\right]\right)=\mathcal{N}\left(\left[\begin{array}{c}
C_{2}^{T} \\
C_{3 a}^{T}
\end{array}\right]\right) .
$$

Hence, there exists an invertible matrix $R_{d}$ such that

$$
\left[\begin{array}{c}
B_{2} \\
B_{3 b}
\end{array}\right]=R_{d}\left[\begin{array}{c}
C_{2}^{T} \\
C_{3 a}^{T}
\end{array}\right] \text {. }
$$

This completes the proof.

The following lemma, which follows directly from the proof of a result presented in [16], [34], gives useful properties of the minimal realization (57) of the SNI transfer function matrix $\bar{G}(s)$.

Lemma 5: (See the proof of Lemma 6 in [34]) Suppose that the transfer function matrix $\bar{G}(s)$, with minimal state space realization (57), is SNI. Then, $\bar{D}=\bar{D}^{T}$, $\operatorname{det}(\bar{A}) \neq 0$ and there exists a matrix $\bar{P}=\bar{P}^{T}>0$ such that

$$
\bar{A} \bar{P}^{-1}+\bar{P}^{-1} \bar{A}^{T} \leq 0 \text { and } \bar{B}=-\bar{A} \bar{P}^{-1} \bar{C}^{T} .
$$

The following lemma is a simple matrix theory result.

Lemma 6: (See e.g., [16]) Given $A \in \mathbb{C}^{n \times n}$ with $j(A-$ $\left.A^{*}\right) \geq 0$ and $B \in \mathbb{C}^{n \times n}$ with $j\left(B-B^{*}\right)>0$, then

$$
\operatorname{det}(I-A B) \neq 0 .
$$

Now, we are in a position to present the proof of Theorem 5.

Proof of Theorem 5: The internal stability of the positivefeedback interconnection between $G(s)$ and $\bar{G}(s)$ will be guaranteed by considering the closed loop system matrix defined in (4) which is given by

$$
\breve{A}=\left[\begin{array}{cc}
A+B \bar{D} C & B \bar{C} \\
\bar{B} C & \bar{A}
\end{array}\right] .
$$

Here, $A, B$ and $C$ are defined as in (54)-(55) and $\bar{A}, \bar{B}, \bar{C}, \bar{D}$ are defined as in (57). To establish internal stability, we show that the matrix $\breve{A}$ is Hurwitz; i.e., all the eigenvalues of $\breve{A}$ lie in the open left-half of the complex plane.

Consider $T=\left[\begin{array}{cc}P-C^{T} \bar{D} C & -C^{T} \bar{C} \\ -\bar{C}^{T} C & \bar{P}\end{array}\right]$ to be a candidate 
Lyapunov matrix, where

$$
P=\left[\begin{array}{cccc}
P_{1} & 0 & 0 & 0 \\
0 & 0 & 0 & 0 \\
0 & 0 & 0 & 0 \\
0 & 0 & 0 & P_{2}
\end{array}\right] \geq 0
$$

$P_{1}>0, P_{2}>0$ are defined as in Lemma 3 and $\bar{P}>0$ is defined as in Lemma 5.

Claim 1: In the case when the matrix $N$ in (59) is negative semidefinite, then $T>0$ if and only if (61) is satisfied. Also, in the case when the matrix $N$ in (59) is positive semidefinite, then $T>0$ if and only if (61) and (62) are satisfied.

To establish this claim, we first note that since $\bar{G}(s)$ is SNI, it follows from Lemma 5 that $\bar{P}$ satisfies (84). This implies that the condition $T>0$ is equivalent to

$$
\begin{aligned}
& P-C^{T} \bar{D} C-C^{T} \bar{C} \bar{P}^{-1} \bar{C}^{T} C>0, \\
\Leftrightarrow & P-C^{T}\left(\bar{D}+\bar{C} \bar{P}^{-1} \bar{C}^{T}\right) C>0, \\
\Leftrightarrow & P-C^{T}\left(\bar{D}-\bar{C} \bar{A}^{-1} \bar{B}\right) C>0 \text { via (84) in Lemma } 5, \\
\Leftrightarrow & P-C^{T} \bar{G}(0) C>0,
\end{aligned}
$$$$
\Leftrightarrow\left[\begin{array}{cccc}
P_{1} & 0 & 0 & 0 \\
0 & 0 & 0 & 0 \\
0 & 0 & 0 & 0 \\
0 & 0 & 0 & P_{2}
\end{array}\right]-\left[\begin{array}{c}
C_{1}^{T} \\
C_{2}^{T} \\
C_{3 a}^{T} \\
C_{3 b}^{T}
\end{array}\right] \bar{G}(0)\left[\begin{array}{llll}
C_{1} & C_{2} & C_{3 a} & C_{3 b}
\end{array}\right]>0 .
$$

Furthermore, using the Schur complement of the LMI in (86), it is straightforward to verify that the condition $T>0$ is equivalent to the conditions

$$
\left[\begin{array}{ll}
-C_{2}^{T} \bar{G}(0) C_{2} & -C_{2}^{T} \bar{G}(0) C_{3 a} \\
-C_{3 a}^{T} \bar{G}(0) C_{2} & -C_{3 a}^{T} \bar{G}(0) C_{3 a}
\end{array}\right]>0
$$

and

$$
\begin{aligned}
& {\left[\begin{array}{cc}
P_{1}-C_{1}^{T} \bar{G}(0) C_{1} & -C_{1}^{T} \bar{G}(0) C_{3 b} \\
-C_{3 b}^{T} \bar{G}(0) C_{1} & P_{3 b}-C_{3 b}^{T} \bar{G}(0) C_{3 b}
\end{array}\right]} \\
& -\left[\begin{array}{cc}
-C_{1}^{T} \bar{G}(0) C_{2} & -C_{1}^{T} \bar{G}(0) C_{3 a} \\
-C_{3 b}^{T} \bar{G}(0) C_{2} & -C_{3 b}^{T} \bar{G}(0) C_{3 a}
\end{array}\right] \\
& \times\left[\begin{array}{ll}
-C_{2}^{T} \bar{G}(0) C_{2} & -C_{2}^{T} \bar{G}(0) C_{3 a} \\
-C_{3 a}^{T} \bar{G}(0) C_{2} & -C_{3 a}^{T} \bar{G}(0) C_{3 a}
\end{array}\right] \\
& \times\left[\begin{array}{ll}
-C_{2}^{T} \bar{G}(0) C_{1} & -C_{2}^{T} \bar{G}(0) C_{3 b} \\
-C_{3 a}^{T} \bar{G}(0) C_{1} & -C_{3 a}^{T} \bar{G}(0) C_{3 b}
\end{array}\right] \\
& >0 .
\end{aligned}
$$

Moreover, (87) is equivalent to

$$
-\left[\begin{array}{c}
C_{2}^{T} \\
C_{3 a}^{T}
\end{array}\right] \bar{G}(0)\left[\begin{array}{ll}
C_{2} & C_{3 a}
\end{array}\right]=-\Xi>0,
$$

where $\Xi$ is defined in (60). This is equivalent to condition (61).
Also, the condition (88) is equivalent to

$$
\begin{aligned}
& {\left[\begin{array}{cc}
P_{1} & 0 \\
0 & P_{2}
\end{array}\right]-\left[\begin{array}{l}
C_{1}^{T} \\
C_{3 b}^{T}
\end{array}\right] \bar{G}(0)\left[\begin{array}{ll}
C_{1} & C_{3 b}
\end{array}\right]} \\
& +\left[\begin{array}{c}
C_{1}^{T} \\
C_{3 b}^{T}
\end{array}\right] \bar{G}(0)\left[\begin{array}{ll}
C_{2} & C_{3 a}
\end{array}\right] \Xi^{-1} \\
& \quad \times\left[\begin{array}{c}
C_{2}^{T} \\
C_{3 a}^{T}
\end{array}\right] \bar{G}(0)\left[\begin{array}{ll}
C_{1} & C_{3 b}
\end{array}\right]>0, \\
& \Leftrightarrow\left[\begin{array}{cc}
P_{1} & 0 \\
0 & P_{2}
\end{array}\right]-\left[\begin{array}{l}
C_{1}^{T} \\
C_{3 b}^{T}
\end{array}\right] N\left[\begin{array}{ll}
C_{1} & C_{3 b}
\end{array}\right]>0 \text { using (59). }
\end{aligned}
$$

This condition is always satisfied in the case where $N$ is negative semidefinite. Hence using (89), we can conclude that $T>0$ if and only if (61) is satisfied in the case when $N$ is negative semidefinite.

Now in the case when $N$ is positive semidefinite, the condition (90) can be rewritten as follows

$$
P_{f}-C_{f}^{T} N C_{f}>0
$$

where $P_{f}=\left[\begin{array}{cc}P_{1} & 0 \\ 0 & P_{2}\end{array}\right]>0$ and $C_{f}=\left[\begin{array}{ll}C_{1} & C_{3 b}\end{array}\right]$. However, using the Schur complement, this is equivalent to the condition

$$
\begin{aligned}
& {\left[\begin{array}{cc}
I & N^{\frac{1}{2}} C_{f} \\
C_{f}^{T} N^{\frac{1}{2}} & P_{f}
\end{array}\right]>0} \\
& \Leftrightarrow I-N^{\frac{1}{2}} C_{f} P_{f}^{-1} C_{f}^{T} N^{\frac{1}{2}}>0 \\
& \Leftrightarrow I-N^{\frac{1}{2}} C_{1} P_{1}^{-1} C_{1}^{T} N^{\frac{1}{2}}-N^{\frac{1}{2}} C_{3 b} P_{2}^{-1} C_{3 b}^{T} N^{\frac{1}{2}}>0 .
\end{aligned}
$$

Now using (80) in Lemma 3, we can define a matrix $M$ as

$$
M=W+L_{1} P_{1}^{-1} C_{1}^{T}
$$

so that

$$
\begin{aligned}
M^{T} M= & C_{2} B_{2}+B_{2}^{T} C_{2}^{T}+C_{3} B_{3}+B_{3}^{T} C_{3}^{T} \\
= & C_{2} B_{2}+B_{2}^{T} C_{2}^{T}+C_{3 a} B_{3 a}+B_{3 a}^{T} C_{3 a}^{T} \\
& +C_{3 b} B_{3 b}+B_{3 b}^{T} C_{3 b}^{T} .
\end{aligned}
$$

Also using (76) in Lemma 3 , we can write $B_{1}$ as

$$
B_{1}=P_{1}^{-1}\left(A_{1}^{T} C_{1}^{T}-L_{1}^{T} W\right) .
$$

Substituting for $W$ in terms of $M$ into this expression for $B_{1}$ gives

$$
\begin{aligned}
B_{1} & =P_{1}^{-1}\left(A_{1}^{T} C_{1}^{T}-L_{1}^{T}\left(M-L_{1} P_{1}^{-1} C_{1}^{T}\right)\right) \\
& =P_{1}^{-1} A_{1}^{T} C_{1}^{T}-P_{1}^{-1} L_{1}^{T} M-P_{1}^{-1} L_{1}^{T} L_{1} P_{1}^{-1} C_{1}^{T} \\
& =-A_{1} P_{1}^{-1} C_{1}^{T}-P_{1}^{-1} L_{1}^{T} M .
\end{aligned}
$$

Also, from the definition of $N$ in (59), it follows that

$$
N\left[C_{2} \quad C_{3 a}\right]=0 .
$$

Therefore, Lemma 4 implies

$$
\begin{aligned}
& N\left[B_{2}^{T} \quad B_{3 b}^{T}\right]=0, \\
& \Rightarrow N\left(C_{2} B_{2}+B_{2}^{T} C_{2}^{T}+C_{3 a} B_{3 a}\right. \\
& \left.+B_{3 a}^{T} C_{3 a}^{T}+C_{3 b} B_{3 b}+B_{3 b}^{T} C_{3 b}^{T}\right) N=0, \\
& \Rightarrow N\left(M^{T} M\right) N=0 \text {, using (92). }
\end{aligned}
$$


Hence, $M N=0$. Therefore,

$$
M N^{\frac{1}{2}}=0 .
$$

Substituting this into (93) implies

$$
\begin{aligned}
& B_{1} N^{\frac{1}{2}}=-A_{1} P_{1}^{-1} C_{1}^{T} N^{\frac{1}{2}}-P_{1}^{-1} L_{1}^{T} M N^{\frac{1}{2}}, \\
\Rightarrow & B_{1} N^{\frac{1}{2}}=-A_{1} P_{1}^{-1} C_{1}^{T} N^{\frac{1}{2}}, \\
\Rightarrow & N^{\frac{1}{2}} C_{1} A_{1}^{-1} B_{1} N^{\frac{1}{2}}=-N^{\frac{1}{2}} C_{1} P_{1}^{-1} C_{1}^{T} N^{\frac{1}{2}} .
\end{aligned}
$$

Substituting (96) into (91) gives the condition

$$
I+N^{\frac{1}{2}} C_{1} A_{1}^{-1} B_{1} N^{\frac{1}{2}}-N^{\frac{1}{2}} C_{3 b} P_{2}^{-1} C_{3 b}^{T} N^{\frac{1}{2}}>0 .
$$

This is equivalent to condition (62). Hence, in the case when $N$ is positive semidefinite, it follows from this and (89) that $T>0$ if and only if conditions (61) and (62) are satisfied. This completes the proof of Claim 1 .

Now, observe that

$$
\begin{aligned}
& T \breve{A}+\breve{A}^{T} T \\
= & {\left[\begin{array}{cc}
P-C^{T} \bar{D} C & -C^{T} \bar{C} \\
-\bar{C}^{T} C & \bar{P}
\end{array}\right] \times\left[\begin{array}{cc}
A+B \bar{D} C & B \bar{C} \\
\bar{B} C & \bar{A}
\end{array}\right] } \\
& +\left[\begin{array}{cc}
A+B \bar{D} C & B \bar{C} \\
\bar{B} C & \bar{A}
\end{array}\right]^{T} \times\left[\begin{array}{cc}
P-C^{T} \bar{D} C & -C^{T} \bar{C} \\
-\bar{C}^{T} C & \bar{P}
\end{array}\right], \\
= & -\left[\begin{array}{cc}
C^{T} \bar{D} W^{T}+L^{T} & C^{T} \bar{W}^{T} \\
\bar{C}^{T} W^{T} & \bar{L}^{T}
\end{array}\right]\left[\begin{array}{cc}
W \bar{D} C+L & W \bar{C} \\
\bar{W} C & \bar{L}
\end{array}\right] \\
\leq & 0 .
\end{aligned}
$$

Together with Claim 1, this implies that $\breve{A}$ has all its eigenvalues in the closed left-half of the complex plane if and only if conditions (61) and (62) are satisfied in the case when $N$ is positive semidefinite; e.g., see Lemma 3.19 in [55]. Similarly, in the case when $N$ is negative semidefinite $\breve{A}$ has all its eigenvalues in the closed left-half of the complex plane if and only if condition (61) is satisfied.

In order to complete the proof of the sufficiency part of the theorem, we must show that if conditions (61) and (62) are satisfied in the case when $N$ is positive semidefinite, then the matrix $\breve{A}$ can have no eigenvalues on the $j \omega$ axis. Similarly, we must show that if conditions (61) and (63) are satisfied in the case that $N$ is negative semidefinite, then the matrix $\breve{A}$ can have no eigenvalues on the $j \omega$ axis.

Indeed, using Lemma 6, the fact that $G(s)$ is NI and the fact that $\bar{G}(s)$ is SNI, we conclude that $\operatorname{det}(I-G(j \omega) \bar{G}(j \omega)) \neq 0$ for all $\omega>0$. This implies that $\breve{A}$ has no eigenvalues on the imaginary axis for $\omega>0$. Thus, to complete the proof, we will show that in the case when $N$ is positive semidefinite, conditions (61) and (62) imply that $\operatorname{det}(\breve{A}) \neq 0$. Similarly, in the case when $N$ is negative semidefinite, we will show that conditions (61) and (63) imply that $\operatorname{det}(\breve{A}) \neq 0$. Indeed,

$$
\begin{aligned}
\operatorname{det}(\breve{A}) & =\operatorname{det}(\bar{A}) \operatorname{det}\left(A+B \bar{D} C-B \bar{C} \bar{A}^{-1} \bar{B} C\right), \\
& =\operatorname{det}(\bar{A}) \operatorname{det}(A+B \bar{G}(0) C) .
\end{aligned}
$$

This implies that $\operatorname{det}(\breve{A}) \neq 0$ if $\operatorname{det}(A+B \bar{G}(0) C) \neq 0$, since $\operatorname{det}(\bar{A}) \neq 0$ using Lemma 5 and the fact that $\bar{G}(s)$ is SNI.
Now, define the matrix

$$
\begin{aligned}
\Lambda & =\left[\begin{array}{cc}
B_{2} \bar{G}(0) C_{2} & B_{2} \bar{G}(0) C_{3 a} \\
B_{3 b} \bar{G}(0) C_{2} & B_{3 b} \bar{G}(0) C_{3 a}
\end{array}\right] \\
& =\left[\begin{array}{c}
B_{2} \\
B_{3 b}
\end{array}\right] \bar{G}(0)\left[\begin{array}{ll}
C_{2} & C_{3 a}
\end{array}\right] .
\end{aligned}
$$

It follows from Lemma 4 that there exists a non-singular matrix $R_{d}$ such that

$$
\Lambda=R_{d}\left[\begin{array}{c}
C_{2}^{T} \\
C_{3 a}^{T}
\end{array}\right] \bar{G}(0)\left[\begin{array}{ll}
C_{2} & C_{3 a}
\end{array}\right]=R_{d} \Xi .
$$

Since the matrix $\Xi$ is assumed to be invertible, this implies that the matrix $\Lambda$ in (99) is invertible.

Now, substituting (55) into (98), it is straightforward to verify that

$$
\begin{aligned}
& \operatorname{det}(A+B \bar{G}(0) C) \\
& =-\operatorname{det} \Lambda \operatorname{det} \\
& \left(\begin{array}{c}
{\left[\begin{array}{cc}
A_{1} & 0 \\
0 & I
\end{array}\right]+\left[\begin{array}{c}
B_{1} \\
B_{3 a}
\end{array}\right]} \\
\times\left(\bar{G}(0)-\bar{G}(0)\left[\begin{array}{ll}
C_{2} & C_{3 a}
\end{array}\right] \Lambda^{-1}\left[\begin{array}{c}
B_{2} \\
B_{3 b}
\end{array}\right] \bar{G}(0)\right) \\
\times\left[\begin{array}{ll}
C_{1} & C_{3 b}
\end{array}\right]
\end{array}\right. \\
& =-\operatorname{det} \Lambda \operatorname{det} \\
& \left(\begin{array}{c}
{\left[\begin{array}{cc}
A_{1} & 0 \\
0 & I
\end{array}\right]+\left[\begin{array}{c}
B_{1} \\
B_{3 a}
\end{array}\right]} \\
\times\left(\bar{G}(0)-\bar{G}(0)\left[\begin{array}{ll}
C_{2} & C_{3 a}
\end{array}\right]\left(R_{d} \Xi\right)^{-1} R_{d}\left[\begin{array}{c}
C_{2}^{T} \\
C_{3 a}^{T}
\end{array}\right] \bar{G}(0)\right) \\
\times\left[\begin{array}{ll}
C_{1} & C_{3 b}
\end{array}\right]
\end{array}\right) \\
& =-\operatorname{det} \Lambda \operatorname{det}\left(\left[\begin{array}{cc}
A_{1} & 0 \\
0 & I
\end{array}\right]+\left[\begin{array}{c}
B_{1} \\
B_{3 a}
\end{array}\right] N\left[\begin{array}{ll}
C_{1} & C_{3 b}
\end{array}\right]\right) \\
& =-\operatorname{det} \Lambda \operatorname{det}\left[\begin{array}{cc}
A_{1} & 0 \\
0 & I
\end{array}\right] \\
& \times \operatorname{det}\left(I+\left[\begin{array}{cc}
A_{1} & 0 \\
0 & I
\end{array}\right]^{-1}\left[\begin{array}{c}
B^{1} \\
B^{3 a}
\end{array}\right] N\left[\begin{array}{ll}
C_{1} & C_{3 b}
\end{array}\right]\right) \\
& =-\operatorname{det} \Lambda \operatorname{det}\left[\begin{array}{cc}
A_{1} & 0 \\
0 & I
\end{array}\right] \\
& \times \operatorname{det}\left(I+\left[\begin{array}{ll}
C_{1} & C_{3 b}
\end{array}\right]\left[\begin{array}{cc}
A_{1} & 0 \\
0 & I
\end{array}\right]^{-1}\left[\begin{array}{c}
B^{1} \\
B^{3 a}
\end{array}\right] N\right) \\
& =-\operatorname{det} \Lambda \operatorname{det}\left[\begin{array}{cc}
A_{1} & 0 \\
0 & I
\end{array}\right] \operatorname{det}\left(I+\left(C_{1} A_{1}^{-1} B_{1}+C_{3 b} B_{3 a}\right) N\right) \text {. }
\end{aligned}
$$

In the case when $N$ is positive semidefinite, (100) and (102) imply that

$$
\begin{aligned}
& \operatorname{det}(A+B \bar{G}(0) C) \\
& =-\operatorname{det} R_{d} \operatorname{det} \Xi \operatorname{det}\left[\begin{array}{cr}
A_{1} & 0 \\
0 & I
\end{array}\right] \\
& \quad \times \operatorname{det}\left[I+\left(C_{1} A_{1}^{-1} B_{1}+C_{3 b} B_{3 a}\right) N^{\frac{1}{2}} N^{\frac{1}{2}}\right], \\
& =-\operatorname{det} R_{d} \operatorname{det} \Xi \operatorname{det}\left[\begin{array}{cc}
A_{1} & 0 \\
0 & I
\end{array}\right] \\
& \quad \times \operatorname{det}\left[I+N^{\frac{1}{2}}\left(C_{1} A_{1}^{-1} B_{1}+C_{3 b} B_{3 a}\right) N^{\frac{1}{2}}\right] .
\end{aligned}
$$


Now using (94), it follows that the columns of the matrix $N^{\frac{1}{2}}$ are contained in the set $\mathcal{N}\left(\left[\begin{array}{c}C_{2}^{T} \\ C_{3 a}^{T}\end{array}\right]\right)$. Hence, it follows from the second part of Lemma 4 that $\left(B_{3 a}+P_{2}^{-1} C_{3 b}^{T}\right) N^{\frac{1}{2}}=0$. This implies that

$$
N^{\frac{1}{2}} C_{3 b} B_{3 a} N^{\frac{1}{2}}=-N^{\frac{1}{2}} C_{3 b} P_{2}^{-1} C_{3 b}^{T} N^{\frac{1}{2}} .
$$

Hence (103) can be written as

$$
\begin{aligned}
\operatorname{det}(A+B \bar{G}(0) C) & -\operatorname{det} R_{d} \operatorname{det} \Xi \operatorname{det}\left[\begin{array}{cc}
A_{1} & 0 \\
0 & I
\end{array}\right] \\
& \times \operatorname{det}\left[I+N^{\frac{1}{2}} C_{1} A_{1}^{-1} B_{1} N^{\frac{1}{2}}-N^{\frac{1}{2}} C_{3 b} P_{2}^{-1} C_{3 b}^{T} N^{\frac{1}{2}}\right], \\
= & -\operatorname{det} R_{d} \operatorname{det} \Xi \operatorname{det}\left[\begin{array}{cc}
A_{1} & 0 \\
0 & I
\end{array}\right] \\
& \times \operatorname{det}\left[I+N^{\frac{1}{2}} C_{1} A_{1}^{-1} B_{1} N^{\frac{1}{2}}-N^{\frac{1}{2}} C_{3 b} P_{3 b}^{-1} C_{3 b}^{T} N^{\frac{1}{2}}\right] .
\end{aligned}
$$

Since the matrices $R_{d}, A_{1}, \bar{A}$ are invertible and also using (61)(62), (98), it follows that $\operatorname{det}(\breve{A}) \neq 0$ as required.

In the case when $N$ is negative semidefinite, we consider the matrix $\tilde{N}=(-N)^{\frac{1}{2}}$. Then (102) implies that

$$
\begin{aligned}
& \operatorname{det}(A+B \bar{G}(0) C) \\
& =-\operatorname{det} R_{d} \operatorname{det} \Xi \operatorname{det}\left[\begin{array}{cr}
A_{1} & 0 \\
0 & I
\end{array}\right] \\
& \quad \times \operatorname{det}\left[I-\tilde{N}\left(C_{1} A_{1}^{-1} B_{1}+C_{3 b} B_{3 a}\right) \tilde{N}\right] .
\end{aligned}
$$

Using (94), it follows that the columns of the matrix $\tilde{N}$ are contained in the set $\mathcal{N}\left(\left[\begin{array}{l}C_{2}^{T} \\ C_{3 a}^{T}\end{array}\right]\right)$. Hence, it follows from the second part of Lemma 4 that $\left(B_{3 a}+P_{2}^{-1} C_{3 b}^{T}\right) \tilde{N}=0$. This implies

$$
\tilde{N} C_{3 b} B_{3 a} \tilde{N}=-\tilde{N} C_{3 b} P_{2}^{-1} C_{3 b}^{T} \tilde{N} .
$$

Hence, (105) can be written as

$$
\begin{aligned}
& -\operatorname{det} R_{d} \operatorname{det} \Xi \operatorname{det}\left[\begin{array}{cr}
A_{1} & 0 \\
0 & I
\end{array}\right] \\
& \quad \times \operatorname{det}\left[I-\tilde{N} C_{1} A_{1}^{-1} B_{1} \tilde{N}+\tilde{N} C_{3 b} P_{2}^{-1} C_{3 b}^{T} \tilde{N}\right], \\
& =-\operatorname{det} R_{d} \operatorname{det} \Xi \operatorname{det}\left[\begin{array}{cr}
A_{1} & 0 \\
0 & I
\end{array}\right] \\
& \quad \times \operatorname{det}\left[I+N^{\frac{1}{2}} C_{1} A_{1}^{-1} B_{1} N^{\frac{1}{2}}-N^{\frac{1}{2}} C_{3 b} P_{3 b}^{-1} C_{3 b}^{T} N^{\frac{1}{2}}\right] .
\end{aligned}
$$

Since the matrices $R_{d}, A_{1}, \bar{A}$ are invertible and also using (61)-(63), (98), it follows that $\operatorname{det}(\breve{A}) \neq 0$ as required. This completes the proof of the sufficiency part of the theorem.

To complete the proof of the necessity part of the theorem, suppose that the positive-feedback interconnection between the NI transfer function matrix $G(s)$ and the SNI transfer function matrix $\bar{G}(s)$ is internally stable. This implies that the matrix $A$ is Hurwitz and hence has all its eigenvalues are in the open left-half of the complex plane. This together with Claim 1 and (97) implies that conditions (61) and (63) are satisfied in the case when $N$ is negative semidefinite. Similarly, in the case when $N$ is positive semidefinite, Claim 1 and (97) implies that conditions (61) and (62) are satisfied. This completes the proof of the theorem.

Proof of Corollary 3: The proof of this corollary will proceeds in an almost identical fashion to the proof of Theorem 5. Indeed, we first state the following claim:

Claim 2: Assume that the matrix $N$ in (59) satisfies $N\left[\begin{array}{ll}C_{1} & C_{3 b}\end{array}\right]=0$, then $T>0$ if and only if (61) is satisfied.

This claim corresponds to Claim 1 in Theorem 5 when we relax the conditions on the matrix $N$. The proof of this claim is similar to the proof of Claim 1 in the proof of Theorem 5 since (90) is automatically satisfied in the case when $N\left[\begin{array}{ll}C_{1} & C_{3 b}\end{array}\right]=0$.

Also, the determinant condition in (98) will be automatically satisfied using the fact $N\left[\begin{array}{ll}C_{1} & C_{3 b}\end{array}\right]=0$ in (101). The proof of the corollary then follows as in the proof of Theorem 5.

Proof of Corollary 4: Note that for the system (54)-(55) corresponding to the case of this corollary, the conditions (61)(63) in Theorem 5 reduce to conditions (65)-(67). Then the proof of the corollary proceeds in an identical fashion to the proof of Theorem 5 for the special case being considered, where the matrix $P$ defined in (85) becomes a matrix of the form $P=\left[\begin{array}{ccc}P_{1} & 0 & 0 \\ 0 & 0 & 0 \\ 0 & 0 & P_{2}\end{array}\right]$.

Proof of Corollary 5: First note that for the system (54)(55) corresponding to the case of this corollary, the conditions (61)-(63) in Theorem 5 reduce to conditions (69)-(71). Then the proof of the corollary proceeds in an identical fashion to the proof of Theorem 5 for the special case being considered, where the matrix $P$ defined in (85) becomes a matrix of the form $P=\left[\begin{array}{cc}P_{1} & 0 \\ 0 & 0\end{array}\right]$

\section{APPENDIX B}

Here, we present the proof of the main results in the paper.

We first show that any NI system can be transformed to the block diagonal form given in (54)-(55).

Lemma 7: Any NI system with transfer function matrix $G(s)$ and minimal state space realization (54), can be transformed to the block diagonal form given in (55).

Proof: Suppose that the transfer function matrix $G(s)$, with a minimal state space realization $\left[\begin{array}{l|l}A & B \\ \hline C & 0\end{array}\right]$ is NI. It follows from Theorem 2.1.1 in [62] that we can find a non-singular state space transformation matrix $T$ such that the matrix $T^{-1} A T$ is in real Jordon block diagonal form and the realization $\left[\begin{array}{c|c}T^{-1} A T & T^{-1} B \\ \hline C T & 0\end{array}\right]$ is minimal. Also, we can choose this transformation so that the Jordon blocks of $T^{-1} A T$ are ordered according to the magnitudes of the corresponding eigenvalues of the matrix $A$, such that the last blocks correspond to the zero eigenvalues of $A$ if they exist. Furthermore, this transformation can be chosen so that 
the Jordan blocks corresponding to the zero eigenvalues are ordered according to increasing order of the Jordan blocks. Also, a further transformation can be applied so that the matrix $A_{3}$ corresponding to the Jordan blocks of order two is of the form (56).

Now, we claim that if $G(s)$ is NI, then there are no Jordan blocks corresponding to zero eigenvalues of order greater than or equal to three. To prove this claim, suppose that there is a Jordon block of $A$ corresponding to a zero eigenvalue of order greater than or equal to three. This together with the minimality of the realization implies that $G_{3}=\lim _{s \rightarrow 0} s^{3} G(s) \neq 0$ which contradicts the NI definition. Thus the zero eigenvalues of $A$ will only have Jordon blocks of order one or two. From this, it now follows that the matrix $T^{-1} A T$ will be of the form (55). This completes the proof of the lemma.

The next lemma is a technical lemma, which will be used in order to prove our results.

Lemma 8: For any full rank matrices $A, B, C$ and $D$ which satisfy $A B=C D$ where $A \in \mathbb{R}^{n \times r}, B \in \mathbb{R}^{r \times n}, C \in$ $\mathbb{R}^{n \times r}, D \in \mathbb{R}^{r \times n}$ and $n \geq r$, there exists an invertible matrix $R$ such that $A=C R$ and $B=R^{-1} D$.

Proof: Since $B$ is of full rank, and $n \geq r, A B=C D$ implies

$$
\begin{aligned}
& A B B^{T}=C D B^{T}, \\
\Rightarrow & A=C D B^{T}\left(B B^{T}\right)^{-1}, \\
\Rightarrow & A=C R
\end{aligned}
$$

where $R=D B^{T}\left(B B^{T}\right)^{-1}$.

To show that $R$ is nonsingular, suppose that $R$ is singular. Then there exists a non-zero $n \times 1$ vector $x$ such that $R x=0$. This implies that $A x=0$ which contradicts the fact that $A$ is a full rank. Hence, that there exists a nonsingular matrix $R$ such that $A=C R$. Also, since $C$ is of full rank and $n \geq r$, it follows that $C$ has a left inverse, which implies that $R B=D$; i.e., $B=R^{-1} D$. This complete the proof.

Proof of Theorem 1: Lemma 7 shows that any strictly proper NI system can be represented in the block diagonal form (54)(55). This implies that we only need to show the equivalence of the assumptions and the conditions (61)-(63) in Theorem 5 and the assumptions and the conditions (13)-(15) in this theorem.

First, it is straightforward to verify that the condition $k \neq 0$ is equivalent to the condition $G_{2} \neq 0$. Also, it follows from (74) and (6) that there exists a full rank matrix $J$ such that

$$
C_{3 a} B_{3 b}=J J^{T} \text {. }
$$

Also, it follows from Lemma 2 and Lemma 8 that there exists an invertible matrix $X$ such that $C_{3 a}=J X$ and $B_{3 b}=X^{-1} J^{T}$. We let $P_{2}=C_{3 a}^{T} B_{3 b}^{T}\left(B_{3 b} B_{3 b}^{T}\right)^{-1}$ and note that $B_{3 b} B_{3 b}^{T}$ is invertible since $B_{3 b}$ is of full rank. Then Lemma 3 implies that $P_{2}$ is symmetric and also we obtain

$$
P_{2}=X^{T} X
$$

In the case when $N$ is positive semidefinite, the definition of $N$ implies that $N\left[\begin{array}{ll}C_{2} & C_{3 a}\end{array}\right]=0$, and hence $N^{\frac{1}{2}}\left[\begin{array}{ll}C_{2} & C_{3 a_{7}}\end{array}\right]=$ 0 . Using (74), it follows that $G_{1}=\left[\begin{array}{ll}C_{2} & C_{3 a}\end{array}\right]\left[\begin{array}{c}B_{2} \\ B_{3 a}\end{array}\right]+$
$C_{3 b} B_{3 b}$, which implies

$$
\begin{aligned}
& N^{\frac{1}{2}} G_{1}=N^{\frac{1}{2}}\left[\begin{array}{ll}
C_{2} & C_{3 a}
\end{array}\right]\left[\begin{array}{c}
B_{2} \\
B_{3 a}
\end{array}\right]+N^{\frac{1}{2}} C_{3 b} B_{3 b}, \\
& \Rightarrow N^{\frac{1}{2}} G_{1}=N^{\frac{1}{2}} C_{3 b} B_{3 b} \text { since } N^{\frac{1}{2}}\left[\begin{array}{ll}
C_{2} & C_{3 a}
\end{array}\right]=0, \\
& \Rightarrow N^{\frac{1}{2}} G_{1} J=N^{\frac{1}{2}} C_{3 b} X^{-1} J^{T} J, \\
& \Rightarrow N^{\frac{1}{2}} G_{1} J\left(J^{T} J\right)^{-1}=N^{\frac{1}{2}} C_{3 b} X^{-1} .
\end{aligned}
$$

Substituting (74), (107) and (108) into condition (62) in Theorem 5, it follows that this condition can be rewritten as

$$
\begin{aligned}
I- & \left(N^{\frac{1}{2}} G_{0} N^{\frac{1}{2}}+N^{\frac{1}{2}} G_{1} J\left(J^{T} J\right)^{-1}\left(J^{T} J\right)^{-T} J^{T} G_{1}^{T} N^{\frac{1}{2}}\right) \\
& >0 .
\end{aligned}
$$

Similarly, in the case when $N$ is negative semidefinite, it follows that $\tilde{N}\left[\begin{array}{ll}C_{2} & C_{3 a}\end{array}\right]=0$. This implies that

$$
\begin{aligned}
& \tilde{N} G_{1}=\tilde{N}\left[\begin{array}{ll}
C_{2} & C_{3 a}
\end{array}\right]\left[\begin{array}{c}
B_{2} \\
B_{3 a}
\end{array}\right]+\tilde{N} C_{3 b} B_{3 b}, \\
& \Rightarrow \tilde{N} G_{1}=\tilde{N} C_{3 b} B_{3 b} \text { since } \tilde{N}\left[\begin{array}{ll}
C_{2} & C_{3 a}
\end{array}\right]=0, \\
& \Rightarrow \tilde{N} G_{1} J=\tilde{N} C_{3 b} X^{-1} J^{T} J \\
& \Rightarrow \tilde{N} G_{1} J\left(J^{T} J\right)^{-1}=\tilde{N} C_{3 b} X^{-1} .
\end{aligned}
$$

Substituting (74), (107) and (110) into condition (63) in Theorem 5, it follows that this condition can be rewritten as

$$
\begin{aligned}
& \operatorname{det}\left(I+\left(\tilde{N} G_{0} \tilde{N}+\tilde{N} G_{1} J\left(J^{T} J\right)^{-1}\left(J^{T} J\right)^{-T} J^{T} G_{1}^{T} \tilde{N}\right)\right) \\
& \neq 0 .
\end{aligned}
$$

Now, using Lemma 1 and substituting for $G_{1}$ and $G_{2}$ from (5) in the Hankel matrix defined in (7), it follows that

$$
\Gamma=\left[\begin{array}{cc}
G_{1} & G_{2} \\
G_{2} & 0
\end{array}\right]=\left[\begin{array}{c}
\tilde{C} \\
\tilde{C} \tilde{A}
\end{array}\right]\left[\begin{array}{ll}
\tilde{B} & \tilde{A} \tilde{B}
\end{array}\right]
$$

where

$$
\begin{aligned}
& \tilde{A}=\left[\begin{array}{cc}
A_{2} & 0 \\
0 & A_{3}
\end{array}\right]=\left[\begin{array}{cc}
0 & 0 \\
0 & A_{3}
\end{array}\right] ; \quad \tilde{B}=\left[\begin{array}{l}
B_{2} \\
B_{3}
\end{array}\right] ; \\
& \tilde{C}=\left[\begin{array}{ll}
C_{2} & C_{3}
\end{array}\right] .
\end{aligned}
$$

Using this and the SVD in (8), it follows that

$$
\left[\begin{array}{c}
\tilde{C} \\
\tilde{C} \tilde{A}
\end{array}\right]\left[\begin{array}{cc}
\tilde{B} & \tilde{A} \tilde{B}
\end{array}\right]=\left[\begin{array}{l}
U_{1} \\
U_{2}
\end{array}\right] V^{T} .
$$

Using Lemma 2 and Lemma 8, it follows that there exists a nonsingular matrix $R$ such that

$$
U=\left[\begin{array}{l}
U_{1} \\
U_{2}
\end{array}\right]=\left[\begin{array}{c}
\tilde{C} \\
\tilde{C} \tilde{A}
\end{array}\right] R=\left[\begin{array}{c}
\hat{C} \\
\hat{C} \hat{A}
\end{array}\right],
$$

where

$$
\begin{array}{r}
\hat{C}=\tilde{C} R, \\
\hat{A}=R^{-1} \tilde{A} R .
\end{array}
$$

This implies that $\hat{A}^{2}=R^{-1} \tilde{A}^{2} R=0$ since $\tilde{A}^{2}=0$. It follows 
that $U \hat{A}=\left[\begin{array}{c}\hat{C} \hat{A} \\ \hat{C} \hat{A}^{2}\end{array}\right]=\left[\begin{array}{c}\hat{C} \hat{A} \\ 0\end{array}\right]=\left[\begin{array}{c}U_{2} \\ 0\end{array}\right]$, which implies

$$
\hat{A}=U^{T} U \hat{A}=U^{T}\left[\begin{array}{c}
U_{2} \\
0
\end{array}\right]=U_{1}^{T} U_{2}
$$

Using this and (9), it follows that

$$
\mathcal{N}(\hat{A})=\operatorname{span}\left\{\hat{V}_{2}\right\}
$$

Also, since $\tilde{A}=\left[\begin{array}{cc}A_{2} & 0 \\ 0 & A_{3}\end{array}\right]=\left[\begin{array}{ccc}0 & 0 & 0 \\ 0 & 0 & I \\ 0 & 0 & 0\end{array}\right]$, it follows that

$$
\mathcal{N}(\tilde{A})=\operatorname{span}\left\{\left[\begin{array}{ll}
I & 0 \\
0 & I \\
0 & 0
\end{array}\right]\right\} \text {. }
$$

Now observe that we can write the matrix $\left[\begin{array}{ll}C_{2} & C_{3 a}\end{array}\right]$ as

$$
\left[\begin{array}{ll}
C_{2} & C_{3 a}
\end{array}\right]=\tilde{C}\left[\begin{array}{cc}
I & 0 \\
0 & I \\
0 & 0
\end{array}\right]
$$

Also, observe that $\hat{A} x=0$ if and only if

$$
\begin{aligned}
& R^{-1} \tilde{A} R x=0 \\
\Leftrightarrow & \tilde{A} R x=0 \\
\Leftrightarrow & R x \in \mathcal{N}(\tilde{A}) .
\end{aligned}
$$

Hence, $\mathcal{N}(\tilde{A})=R \mathcal{N}(\hat{A})$. Therefore it follows from (113) and (114) that

$$
R \operatorname{span}\left\{\hat{V}_{2}\right\}=\operatorname{span}\left\{R \hat{V}_{2}\right\}=\operatorname{span}\left\{\left[\begin{array}{ll}
I & 0 \\
0 & I \\
0 & 0
\end{array}\right]\right\} .
$$

This implies that there exists a nonsingular matrix $\hat{R}$ such that

$$
\left[\begin{array}{ll}
I & 0 \\
0 & I \\
0 & 0
\end{array}\right]=R \hat{V_{2}} \hat{R}
$$

Substituting this into (115) and using (112) implies

$$
\left[\begin{array}{ll}
C_{2} & C_{3 a}
\end{array}\right]=\tilde{C} R \hat{V_{2}} \hat{R}=\hat{C} \hat{V}_{2} \hat{R}=U_{1} \hat{V_{2}} \hat{R}=F \hat{R},
$$

where $F=U_{1} \hat{V}_{2}$ as in (12). Substituting (116) into the matrix (59) and (60) implies that

$$
\begin{aligned}
N & =\left(\bar{G}(0)-\bar{G}(0) F \hat{R}\left(\hat{R}^{T} F^{T} \bar{G}(0) F \hat{R}\right)^{-1} \hat{R}^{T} F^{T} \bar{G}(0)\right) \\
& =\bar{G}(0)-\bar{G}(0) F\left(F^{T} \bar{G}(0) F\right)^{-1} F^{T} \bar{G}(0) \\
& =N_{f}
\end{aligned}
$$

where $N_{f}$ is defined as into (11). Substituting (117) in (109) and (111) implies that conditions (62) and (63) in Theorem 5 are equivalent to conditions (14) and (15) in the theorem respectively.
Also, (116) implies that

$$
\Xi=\left[\begin{array}{c}
C_{2}^{T} \\
C_{3 a}^{T}
\end{array}\right] \bar{G}(0)\left[\begin{array}{ll}
C_{2} & C_{3 a}
\end{array}\right]=\hat{R}^{T} F^{T} \bar{G}(0) F \hat{R} .
$$

It follows that condition (61) in Theorem 5 is equivalent to condition (13) in the theorem since $\hat{R}$ is invertible. This completes the proof of the theorem.

Proof of Corollary 1: In order to prove this corollary, we show that the stability conditions and the assumptions in Corollary 4 are equivalent to the stability conditions and the assumptions in this corollary. First, it is straightforward to verify that the conditions $k \neq 0$ and $n_{2}=0$ are equivalent to the conditions $G_{2} \neq 0$ and $G_{1}=0$. Also, using (74) and the decomposition in (6), it follows that $C_{3 a} B_{3 b}=J J^{T}$, and hence Lemma 8 implies that there exist an invertible matrix $X$ such that $C_{3 a}=J X$. This implies that the matrix $N_{2}$ in (16) is equal to the matrix $N$ in (64). Also, since $C_{3 a}=J X$ and $X$ is invertible, it follows that condition (17) in Corollary 1 is equivalent to condition (65) in Corollary 4. Since $G_{1}=0$, it follows that

$$
\begin{aligned}
& N G_{1}=N\left(C_{3 a} B_{3 a}+C_{3 b} B_{3 b}\right)=0, \\
& \Rightarrow N C_{3 b} B_{3 b}=0, \text { since } N C_{3 a}=0, \\
& \Rightarrow N C_{3 b}=0, \text { since } B_{3 b} \text { is of full rank. }
\end{aligned}
$$

This implies that $N^{\frac{1}{2}} C_{3 b}=0$ in the case when $N$ is positive semidefinite. Using the fact that $G_{0}=-C_{1} A_{1}^{-1} B_{1}$ from Lemma 1, it follows that condition (18) in Corollary 1 is equivalent to condition (66) in Corollary 4. Also, in the case when $N$ is negative semidefinite (118) implies that $\tilde{N} C_{3 b}=0$. Using the fact that $G_{0}=-C_{1} A_{1}^{-1} B_{1}$ from Lemma 1 , it follows that condition (19) in Corollary 1 is equivalent to condition (67) in Corollary 4. This completes the proof of the corollary.

Proof of Theorem 2: In order to prove this theorem, we first show that $\mathcal{N}\left(G_{2}\right) \subseteq \mathcal{N}\left(G_{0}^{T}\right)$ implies the condition $N\left[\begin{array}{ll}C_{1} & C_{3 b}\end{array}\right]=0$ in Corollary 3 , in the case when $G_{1}=0$. Indeed, suppose that $\mathcal{N}\left(G_{2}\right) \subseteq \mathcal{N}\left(G_{0}^{T}\right)$. This implies that

$$
\mathcal{R}\left(G_{2}\right) \supseteq \mathcal{R}\left(G_{0}\right)
$$

where $\mathcal{R}(\cdot)$ denotes the range space of a matrix. Since $G_{2}=$ $J J^{T}$ and $J$ is of full rank, it follows that

$$
\mathcal{R}\left(J J^{T}\right) \supseteq \mathcal{R}\left(G_{0}\right),
$$

which implies that there exist a matrix $Q$ such that $G_{0}=J Q$. Then, we consider the matrix $N$ defined as

$$
N=\bar{G}(0)-\bar{G}(0) C_{3 a}\left(C_{3 a}^{T} \bar{G}(0) C_{3 a}\right)^{-1} C_{3 a}^{T} \bar{G}(0),
$$

which is the formula for the matrix $N$ in Corollary 3 in the case in which $G_{1}=0$. This implies that

$$
N G_{0}=N J Q=0, \text { since } N J=0,
$$

and hence from Lemma 1, it follows that

$$
N C_{1} A_{1}^{-1} B_{1}=0 .
$$

Using a similar calculation as in equation (93) in the proof of 
Theorem 5, this implies that

$$
\begin{aligned}
& N C_{1} A_{1}^{-1}\left(A_{1} P_{1}^{-1} C_{1}^{T}-P_{1}^{-1} L_{1}^{T} M\right) N=0, \\
& \Rightarrow N C_{1} P_{1}^{-1} C_{1}^{T} N=0 \text { using }(95), \\
& \Rightarrow N C_{1}=0 .
\end{aligned}
$$

Also, since $G_{1}=C_{3} B_{3}=C_{3 a} B_{3 a}+C_{3 b} B_{3 b}=0$, it follows that

$$
\begin{aligned}
& N C_{3 a} B_{3 a}+N C_{3 b} B_{3 b}=0, \\
& \Rightarrow N C_{3 b} B_{3 b}=0, \text { since } N C_{3 a}=0, \\
& \Rightarrow N C_{3 b}=0 \text { since } B_{3 b} \text { is of full rank. }
\end{aligned}
$$

Using (119) and (120), it follows that $N\left[\begin{array}{ll}C_{1} & C_{3 b}\end{array}\right]=0$. This implies the assumptions in Corollary 3 are satisfied in the case when $G_{1}=0$. Also, as in the proof of Theorem 1, the condition (17) reduces to condition (61) in Corollary 3.

Proof of Theorem 3: In order to prove this theorem, we show that the stability conditions and the assumptions in this theorem are equivalent to the stability conditions and the assumptions in Corollary 5. First, it is straightforward to verify that the conditions $n_{2} \neq 0$ and $k=0$ are equivalent to the conditions $G_{1} \neq 0$ and $G_{2}=0$. Using Lemma 8 and the fact that $G_{1}=C_{2} B_{2}$ from Lemma 1, it follows that there exists an invertible matrix $R$ such that $C_{2}=F_{1} R$, where the matrix $F_{1}$ is given in (20). This implies that the matrix $N_{1}$ in (21) is equal to the matrix $N$ in (68). Also, since $C_{2}=F_{1} R$ and $R$ is invertible, it follows that condition (22) in this theorem is equivalent to condition (69) in Corollary 5. Finally, using the fact that $G_{0}=-C_{1} A_{1}^{-1} B_{1}$ from Lemma 1 , it follows that conditions (23) and (24) in this theorem are equivalent to conditions (70) and (71) in Corollary 5 respectively. This completes the proof of the theorem.

Proof of Theorem 4: In order to prove this theorem, we first show that $\mathcal{N}\left(G_{1}^{T}\right) \subseteq \mathcal{N}\left(G_{0}^{T}\right)$ implies the condition $N\left[\begin{array}{ll}C_{1} & C_{3 b}\end{array}\right]=0$ in Corollary 3 , in the case when $G_{2}=0$. Indeed, suppose that $\mathcal{N}\left(G_{1}^{T}\right) \subseteq \mathcal{N}\left(G_{0}^{T}\right)$. This implies that

$$
\mathcal{R}\left(G_{1}\right) \supseteq \mathcal{R}\left(G_{0}\right) \text {. }
$$

Since $G_{1}=C_{2} B_{2}$ from Lemma 1 and $B_{2}$ is of full rank using Lemma 2 , it follows that $\mathcal{R}\left(C_{2}\right)=\mathcal{R}\left(G_{1}\right)$. Using (121), it follows that

$$
\mathcal{R}\left(C_{2}\right) \supseteq \mathcal{R}\left(G_{0}\right),
$$

which implies that there exists a matrix $Q$ such that $G_{0}=$ $C_{2} Q$. Then, we consider the matrix $N$ defined as

$$
N=\bar{G}(0)-\bar{G}(0) C_{2}\left(C_{2}^{T} \bar{G}(0) C_{2}\right)^{-1} C_{2}^{T} \bar{G}(0),
$$

which is the formula for the matrix $N$ in Corollary 3 for the case in which $G_{2}=0$. This implies that

$$
N G_{0}=N C_{2} Q=0, \text { since } N C_{2}=0
$$

and hence from Lemma 1 it follows that

$$
N C_{1} A_{1}^{-1} B_{1}=0 \text {. }
$$

Using a similar calculation as in equation (93) in the proof of Theorem 5, this implies

$$
\begin{aligned}
& N C_{1} A_{1}^{-1}\left(A_{1} P_{1}^{-1} C_{1}^{T}-P_{1}^{-1} L_{1}^{T} M\right) N=0, \\
& \Rightarrow N C_{1} P_{1}^{-1} C_{1}^{T} N=0, \text { using (95) } \\
& \Rightarrow N C_{1}=0 .
\end{aligned}
$$

This implies the assumptions in Corollary 3 are satisfied in the case when $G_{2}=0$. Also, as in the proof of Theorem 1, condition (22) reduces to condition (61) in Corollary 3.

Proof of Corollary 2: In the case where $G_{1}$ is assumed to be invertible in this corollary, it follows that the matrix $F_{1}$ in (20) is invertible. Then, the condition (22) reduces to the condition $\bar{G}(0)<0$ and the corollary follows immediately from Corollary 4 . In the case where $G_{2}$ is assumed to be positive definite in this corollary, it follows that the matrix $J$ in (6) is invertible. Then, the condition (22) reduces to the condition $\bar{G}(0)<0$ and the corollary follows immediately from Corollary 2. 